\title{
ON A FAMILY OF $(1,7)$-POLARISED ABELIAN SURFACES
}

\author{
ALFIO MARINI*
}

\begin{abstract}
We study the geometry of the moduli space of $(1,7)$-polarised abelian surfaces with canonical level structure in detail. In particular we describe the locus where the syzygies of the embedded Heisenberg-invariant abelian surface degenerate, and relate this to the other known descriptions of the moduli space in question.
\end{abstract}

\section{Introduction}

The starting point for this article is the paper by Manolache and Schreyer [16], where the authors find a birational model of the moduli space of $(1,7)$-polarised abelian surfaces with canonical level structure, namely a Fano 3-fold of genus 12 called $V_{22}$. They study the locally free resolution of a $(1,7)$-polarised abelian surface $A \subset \mathrm{P}^{6}(V)$, where $V \cong H^{0}(A, \mathscr{O}(1))^{\prime}$. As we shall see, $\mathrm{SL}_{2}\left(\mathrm{Z}_{7}\right)$ acts on $V$, and therefore on these surfaces and on their resolutions. Every such resolution determines a twisted cubic curve in $\mathrm{P}^{3}(U)$, where $U$ is a certain 4-dimensional irreducible $\mathrm{SL}_{2}\left(\mathrm{Z}_{7}\right)$-module. The set of such curves consists of those annihilated by the unique $\mathrm{SL}_{2}\left(\mathrm{Z}_{7}\right)$-invariant net of quadrics $\Delta \subset S^{2} U$ and the moduli space $H(\Delta)$ of such curves is isomorphic to $V_{22}$.

The other fundamental paper on which this article is based is [8] by Gross and Popescu, where the authors also show that the moduli space of $(1,7)$ polarised abelian surfaces with canonical level structure is birational to $V_{22}$, but using a different approach and consequently a different model. Their model is the variety of sum of powers $\operatorname{VSP}(Q, 6)$ that parametrises polar hexagons to the Klein quartic $Q \subset \mathrm{P}^{2}(W)$, where $W$ is a 3-dimensional irreducible $\mathrm{SL}_{2}\left(\mathrm{Z}_{7}\right)$-module and $Q$ is the unique invariant curve of minimal degree 4 (see Appendix B). Such a polar hexagon is a configuration of six points in $\mathrm{P}^{2}\left(W^{\prime}\right)$, where $W^{\prime}$ is the dual of $W$, and $\mathrm{P}^{2}\left(W^{\prime}\right)$ is naturally contained in $\mathrm{P}^{6}(V)$ as the projectivisation of the +1 -eigenspace of a certain involution $\iota: V \rightarrow V$.

\footnotetext{
* This article is a version of my University of Bath Ph.D. thesis [17], written under the supervision of Greg Sankaran and funded by a scholarship from INdAM. I am grateful to Alastair King, Klaus Hulek and Mark Gross, whose comments contributed to this paper.
}

Received August 8, 2003. 
What Gross and Popescu show in [8] is that, for a general $A$, these six points are precisely the odd 2-torsion points of $A$, and that each point determines a Calabi-Yau 3-fold containing $A$. More precisely $A$ is defined as the intersection of those six 3 -folds. Notice that $Q$ is a model of $X(7)$, the modular curve of level 7, and that in [8] the latter notation is used in place of $Q$. However the curve $Q^{\prime} \subset \mathrm{P}^{2}\left(W^{\prime}\right)$ (also the unique invariant quartic and also isomorphic to $X(7))$ will also play a role in this article and we will be careful to distinguish the two.

For the case of (1,5)-polarised abelian surfaces, Horrocks and Mumford [10] showed that a birational model of the moduli space is $P^{3}$. In a more detailed analysis, Barth, Hulek and Moore [1] showed that in fact every point of $\mathrm{P}^{3}$ determines a surface and they also described the subvariety of $\mathrm{P}^{3}$ that parametrises degenerate abelian surfaces, that is the boundary of the moduli space. Furthermore they gave a precise description of all the degenerations. A natural question raised by [16] and [8] is, can a similar analysis be made for $V_{22}$ ? This question is approached by Melliez and Ranestad [18], who find degenerations similar to those occurring in [1].

While bearing this in mind, we take a slightly different point of view and examine the cases where the construction of [16] degenerates, studying the subvariety $B \subset H(\Delta)$ which parametrises degenerate twisted cubic curves. We show that all points in $B$ determine surfaces in $\mathrm{P}^{6}(V)$ and we find the corresponding elements of $\operatorname{VSP}(Q, 6)$. Our original expectation was that $B$ would also be the subvariety which parametrises degenerate abelian surfaces, and indeed $B$ is birational to the Kummer modular surface that parametrises translation scrolls (the most general degenerations arising in [18] and [1]). Nevertheless, $B$ does not in fact parametrise the translation scrolls and so cannot be the whole boundary of the moduli space of $(1,7)$-polarised abelian surfaces with canonical level structure. In fact, it follows from our description and the results of [18] that the surface parametrised by a general point of $B$ is smooth. However, in this article we will still speak of $B$ as the "boundary" in the sense that it parametrises degenerate twisted cubic curves and will consider that the corresponding abelian surfaces are still, in some sense, degenerate. Indeed they are not general in the sense of [8]. The more special degenerations occur both in our locus and the locus considered by Melliez and Ranestad, who found them independently of this work.

\subsection{The structure of this article}

Our article is divided into five sections. In Section 1 we introduce the subject of abelian varieties, then the crucial action of the Heisenberg group, which is the key to handling these varieties. Finally we report all the key results we need from [16] and [8] that are going to define the starting point of the following sec- 
tions. Most of the notations are thereafter consistent with those papers. Among others, especially important are the 4-dimensional -1-eigenspace $U^{\prime}:=V_{-}$ of the involution $\iota$, which is dual to $U$. Notice that $S^{2} U^{\prime}=L \oplus W^{\prime}$ where $L=\Delta^{\perp}$. But also $S^{3} W^{\prime}=L \oplus W$ and hence we have an incomplete linear system of cubics on $\mathrm{P}^{2}\left(W^{\prime}\right)$ which gives an embedding $\mathrm{P}^{2}\left(W^{\prime}\right) \hookrightarrow \mathrm{P}^{6}(L)$.

In Section 2 we view $H(\Delta)$ as an orbit space, specifically the space $M_{\Delta}$ of $3 \times 2$ matrices with entries in the 4-dimensional space $U^{\prime}$ and with the condition $\Delta$ on the minors (that is, the minors are in $L$ ), and obvious actions of $\operatorname{GL}(3, C)$ and $\operatorname{GL}(2, C)$. The description of the semi-stable points of this space leads to Proposition 3.4, which says that the quotient space $M_{\Delta}^{s} / \sim$ and $H(\Delta)$ are isomorphic. Furthermore we show that $B$ may be also described as the locus of orbits which contain a representative matrix with at least one zero entry. This leads to Proposition 3.8, which says that the locus $B$ in $H(\Delta)$ is birational to $\Gamma \times \mathrm{P}^{1} \cong Q \times \mathrm{P}^{1}$, where $\Gamma \subset \mathrm{P}^{3}\left(U^{\prime}\right)$ is a curve naturally isomorphic to $Q$.

Note that the modular curve of level 7 has 24 cusps corresponding to the 24 flexes in the models $Q$ and $Q^{\prime}$. Throughout this article we are going to use the word "cusp" also for the images of the cusps of $X(7)$ in $Q, Q^{\prime}$ and $\Gamma$.

At the end of Section 2 we also report several results from [21], describing in more detail three different models of the Fano 3-fold $V_{22}$, two of which $(H(\Delta)$ and $\operatorname{VSP}(Q, 6))$ we have introduced already.

We start Section 3 by presenting several results from [10] that are going to be used for comparisons in the sequel. In this way we can recover more information about the features of surfaces parametrised by boundary points of $H(\Delta)$. To present an example of this sort of study, we also describe the work in $[1]$ on the $(1,5)$ case (Section 4$)$.

Section 5 is divided into four subsections. In the first one we prove several results, among them Proposition 5.4: that to every point of the locus $B$ in $H(\Delta)$ it is possible to associate a surface.

The second subsection, devoted to general degenerations, contains several side results and culminates in Proposition 5.12, which relates degenerate twisted cubic curves and the first degeneration of a set of six points in $\operatorname{VSP}(Q, 6)$ : where three of the six points lie on the Hessian of the Klein quartic $\operatorname{Hess}\left(Q^{\prime}\right) \subset \mathrm{P}^{2}\left(W^{\prime}\right)$. Furthermore, the images of these three points under the embedding $\mathrm{P}^{2}\left(W^{\prime}\right) \hookrightarrow \mathrm{P}^{6}(L)$ are collinear and the intersection of the three associated Calabi-Yaus is a 3 -fold $\mathcal{U}_{a}$ of degree 7 . Thus the configuration of six points is not general in the sense of [8] (see Proposition 2.9 below). The most general surface related to such a degeneration is then $\mathscr{U}_{a}$ intersected with any Calabi-Yau defined by any of the other three points.

The surfaces in the third subsection (parametrised by a subvariety $B^{\prime} \subset B$ ) are relatively simple to describe, because they are defined by the intersection 
TABLE 1. Hierarchy of the degenerations

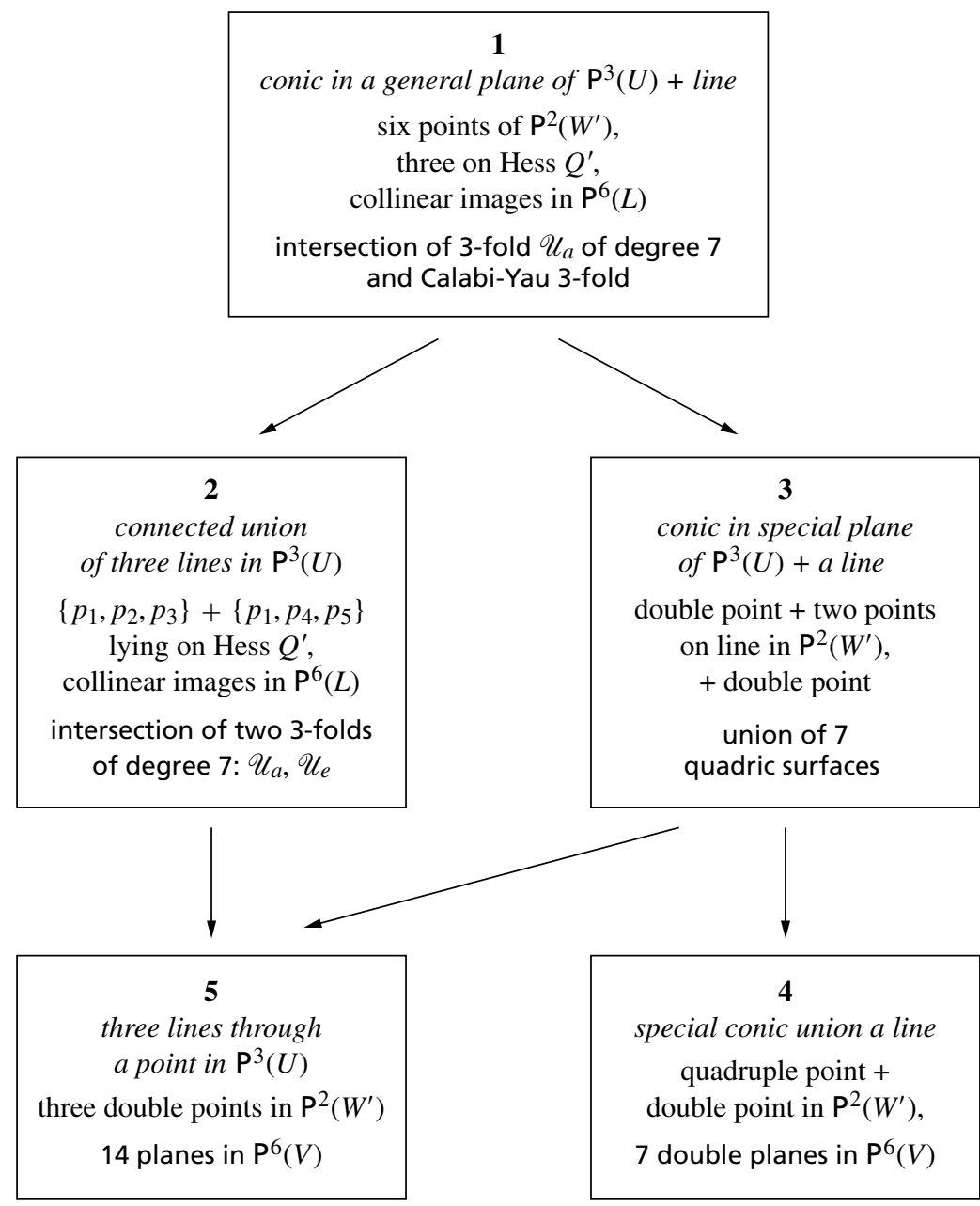

of two 3 -folds $\mathscr{U}_{a}$ and $\mathscr{U}_{e}$ of degree 7 as above. There are two sets of three points as above with one point in common, and the degenerate twisted cubic curve associated is the connected union of three lines. This is summarised in Proposition 5.14.

Further degenerations of $(1,7)$-polarised abelian surfaces arise over the cusps of $\Gamma$, and are studied in the fourth subsection. Proposition 5.16 tells that one gets three types of reducible surfaces: 7 quadric surfaces, each contained in some $\mathrm{P}^{3} \subset \mathrm{P}^{6}(V)$, or 7 double planes in $\mathrm{P}^{6}(V)$, or 14 planes in $\mathrm{P}^{6}(V)$. We work out the related degenerations of $H(\Delta)$ and $\operatorname{VSP}(Q, 6)$ as well. Notice 
that in the $(1,5)$ case there is no configuration analogous to the 14 planes (see Remark 5.19).

All the results proved in this article are presented in the following comprehensive main theorem which lists and classifies the surfaces (possibly degenerations of smooth $(1,7)$-polarised abelian surfaces) parametrised by $B \subset H(\Delta)$ :

Theorem A. Let $[\alpha] \in B \subset H(\Delta) \cong \operatorname{VSP}(Q, 6)$. Then $[\alpha]$ determines:

i. a singular twisted cubic curve $C_{\alpha}$ in $\mathrm{P}^{3}(U)$,

ii. six points in $\mathrm{P}^{2}\left(W^{\prime}\right)$ (more precisely, a 0 -dimensional subscheme $\Xi \subset$ $\mathrm{P}^{2}\left(W^{\prime}\right)$ of length 6$)$, and

iii. a surface $A_{\alpha} \subset \mathrm{P}^{6}(V)$, as follows.

1. If $[\alpha] \in B \backslash B^{\prime}$ and $[\alpha]$ is not over a cusp of $\Gamma$, then

i. $C_{\alpha}$ is a smooth conic in a general plane of $\mathrm{P}^{3}(U)$ union a line;

ii. $\Xi$ is six points of $\mathrm{P}^{2}\left(W^{\prime}\right)$, three of which lie on Hess $\left(Q^{\prime}\right)$, whose images in $\mathrm{P}^{6}(L)$ are collinear;

iii. $A_{\alpha}$ is the intersection of a 3-fold $\mathscr{U}_{a}$ of degree 7 determined by the collinear points, and any Calabi-Yau 3-fold determined by any of the remaining ones.

2. If $[\alpha] \in B^{\prime}$ and $[\alpha]$ is not over a cusp of $\Gamma$, then

i. $C_{\alpha}$ is the connected union of three lines in $\mathrm{P}^{3}(U)$;

ii. $\Xi$ is six points of $\mathrm{P}^{2}\left(W^{\prime}\right)$ with $\left\{p_{1}, p_{2}, p_{3}\right\}$ and $\left\{p_{1}, p_{4}, p_{5}\right\}$ lying on Hess $\left(Q^{\prime}\right)$, whose images in $\mathrm{P}^{6}(L)$ form two sets of three collinear points of $\mathrm{P}^{6}(L)$;

iii. $A_{\alpha}$ is the intersection of two 3-folds of degree 7: $\mathscr{U}_{a}$ determined by the first set of collinear points, and $\mathscr{U}_{e}$ determined by the second set of collinear points.

3. If $[\alpha]$ is general over a cusp of $\Gamma$, then

i. $C_{\alpha}$ is a smooth conic in a special plane (defined by the cusp) of $\mathrm{P}^{3}(U)$ union a line,

ii. $\Xi$ is a double point and two single points on a line in $\mathrm{P}^{2}\left(W^{\prime}\right)$, plus a second double point: both the double points are in $Q \cap \operatorname{Hess}\left(Q^{\prime}\right)$, i.e. at cusps;

iii. $A_{\alpha}$ is the union of 7 quadric surfaces, each contained in some $\mathrm{P}^{3} \subset$ $\mathrm{P}^{6}(V)$.

4. If $[\alpha]$ is special over a cusp of $\Gamma$ as in Proposition 5.16, part (2a), then

i. $C_{\alpha}$ is a smooth special conic union a line;

ii. $\Xi$ is a quadruple point plus a double point in $\mathrm{P}^{2}\left(W^{\prime}\right)$, both at cusps; 
iii. $A_{\alpha}$ is the union of 7 double planes in $\mathrm{P}^{6}(V)$.

5. If $[\alpha] \in B^{\prime}$ is over a cusp of $\Gamma$, then

i. $C_{\alpha}$ is three lines through a point in $\mathrm{P}^{3}(U)$;

ii. $\Xi$ is three double points in $\mathrm{P}^{2}\left(W^{\prime}\right)$, all at cusps;

iii. $A_{\alpha}$ is the union of 14 planes in $\mathrm{P}^{6}(V)$.

Proof. See Proposition 2.6, Proposition 2.9, Proposition 5.12, Proposition 5.14 and Proposition 5.16.

We illustrate the results of the main theorem and the degenerations that occur among the cases in Table 1.

\section{Moduli spaces}

\subsection{Moduli spaces of abelian varieties}

For general background on moduli of abelian varieties and level structures we refer to [12, Section I.1].

Recall that a polarisation on an abelian variety $A$ is the class in NS $(A)$ of an ample line bundle $\mathscr{L}$. If $A=\mathrm{C}^{g} / L$, where $L \subset \mathrm{C}^{g}$ is a cocompact lattice, then we may regard a polarisation as a Riemann form, that is, a positive definite hermitian form $H$ on $\mathrm{C}^{g}$ whose imaginary part $H^{\prime}=\operatorname{Im}(H)$ is integer-valued on $L$.

A line bundle $\mathscr{L}$ representing a polarisation defines a map $\lambda: A \rightarrow \hat{A}=$ $\operatorname{Pic}^{0} A$ by $\lambda(x)=t_{x}^{*} \mathscr{L} \otimes \mathscr{L}^{-1}$, where $t_{x}$ is translation by $x$. The map $\lambda$ depends only on the polarisation, not on the choice of the line bundle $\mathscr{L}:$ we define $K(\mathscr{L})=$ ker $\lambda$. A canonical level structure is a symplectic isomorphism $K(\mathscr{L}) \stackrel{\sim}{\rightarrow}\left(\mathrm{Z}_{e_{1}} \times \cdots \times \mathrm{Z}_{e_{g}}\right)^{2}$, where $\left(e_{1}, \ldots, e_{g}\right)$ is the type of the polarisation (the elementary divisors of $\left.H^{\prime}\right)$. In our case $g=2$ and $\left(e_{1}, e_{2}\right)=(1,7)$. If $x$, $y \in K(\mathscr{L})$, then the skew-symmetric (Weil) pairing induced on $K(\mathscr{L})$ by $H^{\prime}$ is given by $e^{\mathscr{L}}(x, y)=\exp \left(2 \pi i H^{\prime}(x, y)\right)$.

\subsection{The Heisenberg group}

For general facts about Heisenberg groups see [14]: for details of our case, see [7] and [8].

The Heisenberg group $H_{7}$ is generated by two elements $\sigma$ and $\tau$ : via the Schrödinger representation they act on a basis of $\mathrm{P}\left(H^{0}(\mathscr{L})^{\prime}\right)$ (given by canonical theta functions, see [14]) by

$$
\sigma\left(x_{i}\right)=x_{i-1}, \quad \tau\left(x_{i}\right)=\xi^{-i}\left(x_{i}\right),
$$

where $\xi=e^{2 \pi i / 7}$. Thus the image of $A$ in $\mathrm{P}\left(H^{0}(\mathscr{L})^{\prime}\right)$ is invariant under the action of the Heisenberg group via the Schrödinger representation. In particular, 
if $A$ is embedded this way in $\mathrm{P}\left(H^{0}(\mathscr{L})^{\prime}\right)$, then $H^{0}\left(\mathscr{I}_{A}(n)\right)$ is also a representation of the Heisenberg group. Since $[\sigma, \tau]=\xi$, the Heisenberg group is a central extension

$$
1 \longrightarrow \mu_{7} \longrightarrow H_{7} \longrightarrow \mathrm{Z}_{7} \times \mathrm{Z}_{7} \longrightarrow 0 .
$$

$K(\mathscr{L})$ can be viewed as a subgroup of the automorphism group of $A$ via translations, and the order 2 subgroup $\pm 1_{A}$ acts on $K(\mathscr{L})$ by inner automorphisms. The Schrödinger representation of the extended Heisenberg group $G_{7}$ is defined by $G_{7}=H_{7} \rtimes\langle\iota\rangle$, where $\iota$ acts on $H_{7}$ via $\iota\left(\xi, x_{1}, x_{2}\right)=\left(\xi,-x_{1},-x_{2}\right)$, and $\iota$ acts on $V$ by $\iota\left(x_{i}\right)=-x_{-i}$. Note that our $\iota$ here is $-\iota$ in [7] and [8].

$\iota$, acting as an involution on $V$, has two eigenspaces $V_{ \pm}$, with eigenvalues \pm 1 . We will write $\mathbf{P}_{+}=\mathbf{P}\left(V_{+}\right) \subseteq \mathbf{P}(V)$ and similarly $\mathbf{P}_{-}=\mathbf{P}\left(V_{-}\right)$. In the $(1,7)$ case, $\mathrm{P}_{-}$is given by the equations $x_{i}=x_{-i}$ for $i \in \mathrm{Z}_{7}$ and $\mathrm{P}_{+}$is given by the equations $x_{i}=-x_{-i}$ for $i \in \mathrm{Z}_{7}$.

Let $N\left(H_{7}\right)$ be the normaliser of the Heisenberg group $H_{7}$ inside $\operatorname{SL}(V)$, where the inclusion $H_{7} \hookrightarrow \mathrm{SL}(V)$ is via the Schrödinger representation. We have a sequence of inclusions

$$
Z\left(H_{7}\right)=\mu_{7} \subseteq H_{7} \subseteq N\left(H_{7}\right),
$$

and it is easy to see that $N\left(H_{7}\right) / H_{7}=\mathrm{SL}_{2}\left(\mathrm{Z}_{7}\right)$, in fact $N\left(H_{7}\right)$ is a semidirect product $H_{7} \rtimes \mathrm{SL}_{2}\left(Z_{7}\right)$. Therefore the Schrödinger representation of $H_{7}$ induces a 7-dimensional representation $\rho_{7}: \mathrm{SL}_{2}\left(Z_{7}\right) \rightarrow \mathrm{SL}(V)$. The spaces $V_{+}$and $V_{-}$are both invariant under $\rho_{7}$, and $\rho_{7}$ splits as $\rho_{+} \oplus \rho_{-}$, where $\rho_{ \pm}$ is the representation of $\mathrm{SL}_{2}\left(\mathrm{Z}_{7}\right)$ acting on $V_{ \pm}$. Note that $\rho_{+}$is trivial on the centre of $\mathrm{SL}_{2}\left(Z_{7}\right)$, so in fact it descends to give an irreducible representation $\rho_{+}: \mathrm{PGL}_{2}\left(\mathrm{Z}_{7}\right) \rightarrow \mathrm{GL}\left(V_{+}\right)$. In the beautiful treatise [13] by Felix Klein, the polynomial invariants of this representation are computed and the quartic ${ }^{1}$

$$
f_{\text {Klein }}^{\prime}=y_{1}^{3} y_{2}+y_{2}^{3} y_{3}+y_{3}^{3} y_{1}
$$

is the unique invariant of minimal degree 4 . The smooth quartic curve defined by this invariant

$$
Q^{\prime}=\left\{f_{\text {Klein }}^{\prime}=0\right\} \subset \mathrm{P}_{+}^{2}
$$

is an isomorphic image of the modular curve of level 7, and has $\mathrm{PGL}_{2}\left(\mathrm{Z}_{7}\right)$ as its full automorphism group.

Throughout we use the notation $W^{\prime}:=V_{+}$and $U^{\prime}:=V_{-}$, and we are going to work with their dual spaces as well, which as $\mathrm{SL}_{2}\left(\mathrm{Z}_{7}\right)$-modules are not

\footnotetext{
${ }^{1}$ The notation $f_{\text {Klein }}^{\prime}$ is chosen because in Appendix B we work with a quartic given by the same equation, but embedded in the dual space $\mathrm{P}_{+}^{2^{\prime}}$, which is not isomorphic to $\mathrm{P}_{+}^{2}$ as a $\mathrm{SL}_{2}\left(\mathrm{Z}_{7}\right)$-module.
} 
isomorphic to them. More information about the representations of $\mathrm{SL}_{2}\left(\mathrm{Z}_{7}\right)$ and these spaces can be found in subsection A.3 in Appendix A.

\subsection{The moduli space of $(1,7)$-polarised abelian surfaces}

In this subsection we briefly recall the main results by Manolache and Schreyer [16] about the moduli space of $(1,7)$-polarised abelian surfaces with canonical level structure. A central result is the following

Theorem 2.1 ([16], Theorem 3.2). An abelian surface A, $G_{7}$-invariantly embedded in $\mathrm{P}^{6}(V)$, has a $G_{7}$-invariant resolution of the form

$$
\begin{aligned}
0 \longleftarrow \mathscr{O}_{A} \longleftarrow \mathcal{O} \longleftarrow & \beta \\
\longleftarrow & V_{4} \mathcal{O}(-3) \stackrel{\alpha}{\longleftarrow} 2 S \Omega^{3} \\
& \stackrel{\alpha^{\prime}}{\longleftarrow} 3 V_{1} \mathcal{O}(-4) \stackrel{\beta^{\prime}}{\longleftarrow} \mathcal{O}(-7) \longleftarrow 0
\end{aligned}
$$

with $\alpha^{\prime}=\left(\begin{array}{rr}0 & 1 \\ -1 & 0\end{array}\right){ }^{t} \alpha$ and $\beta^{\prime}={ }^{t} \beta$.

Note that all the modules in the above sequence are $G_{7}$-modules, and $S$ is the non-trivial character of $\langle\iota\rangle$. See Appendix A for the table of characters of $G_{7}$.

Proposition 2.2 ([16], Proposition 3.3). $\alpha$ has entries in a 4-dimensional vector space (see Appendix A): more precisely

$$
\operatorname{Hom}_{G_{7}}\left(S \Omega^{3}, V_{4} \mathscr{O}(-3)\right)=4 I .
$$

A key observation is the following.

REMARK 2.3 ([16], Remark 3.4). Let $\mathscr{F}_{1}$ and $\mathscr{F}_{2}$ be two $G_{7}$-sheaves. Then $\operatorname{Hom}_{G_{7}}\left(\mathscr{F}_{1}, \mathscr{F}_{2}\right)$ is a $N$-module, because $G_{7}=H_{7} \rtimes Z_{2}$ is a normal subgroup

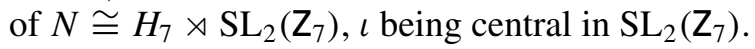

Using the character table of $\mathrm{SL}_{2}\left(Z_{7}\right)$ and with the notation from Appendix A, we see that

$$
\operatorname{Hom}\left(\Omega^{3}, \mathscr{O}(-3)\right) \cong \operatorname{Hom}\left(\mathscr{O}(-4), \Omega^{3}\right)=\wedge^{3} V=V_{1} \oplus 4 V_{1}^{\#}, \quad \text { and }
$$

$\operatorname{Hom}_{G_{7}}\left(S \Omega^{3}, V_{4} \mathscr{O}(-3)\right) \cong \operatorname{Hom}_{G_{7}}\left(V_{1} \mathcal{O}(-4), S \Omega^{3}\right)=U^{\prime}:=\left\langle u_{0}, u_{1}, u_{2}, u_{3}\right\rangle$.

If we equip $V$ with the canonical basis $\left\langle e_{0} \ldots e_{6}\right\rangle$, then we get that the elements of $\mathrm{Hom}_{G_{7}}\left(V_{1} \mathcal{O}(-4), S \Omega^{3}\right)$ are given by the following $1 \times 7$ matrices with entries in $\wedge^{3} V$ :

$$
\begin{aligned}
& u_{0}=\left(e_{k+1} \wedge e_{k+4} \wedge e_{k+2}-e_{k+6} \wedge e_{k+3} \wedge e_{k+5}\right)_{k \in \mathrm{Z}_{7}}, \\
& u_{1}=\left(e_{k} \wedge e_{k+1} \wedge e_{k+6}\right)_{k \in \mathrm{Z}_{7}}, \\
& u_{2}=\left(e_{k} \wedge e_{k+2} \wedge e_{k+5}\right)_{k \in \mathrm{Z}_{7}} \\
& u_{3}=\left(e_{k} \wedge e_{k+4} \wedge e_{k+3}\right)_{k \in \mathrm{Z}_{7}} .
\end{aligned}
$$


Finally $\operatorname{Hom}_{G_{7}}\left(V_{1}^{\#} \mathcal{O}(-4), S \Omega^{3}\right)=\left\langle u_{0}^{\#}\right\rangle$, where

$$
u_{0}^{\#}=\left(e_{k+1} \wedge e_{k+4} \wedge e_{k+2}+e_{k+6} \wedge e_{k+3} \wedge e_{k+5}\right)_{k \in Z_{7}} .
$$

Furthermore, the elements of $\operatorname{Hom}_{G_{7}}\left(S \Omega^{3}, V_{4} \mathcal{O}(-3)\right)$ are given by the transposes of the above matrices. Notice also that the composition of $\operatorname{Hom}(\mathscr{O}(-4)$, $\left.\Omega^{3}\right)$ and $\operatorname{Hom}\left(\Omega^{3}, \mathscr{O}(-3)\right)$ is given by the wedge product, if we identify canonically $\wedge{ }^{6} V$ with $V^{*}=V_{3}=H^{0}(\mathscr{O}(1))$. A direct computation gives us the non-zero compositions:

$$
\begin{aligned}
\lambda:=u_{0}^{t} u_{2}=u_{2}^{t} u_{0}=-u_{3}^{t} u_{3}= & \left(\begin{array}{ccccccc}
0 & 0 & x_{1} & 0 & 0 & -x_{6} & 0 \\
0 & 0 & 0 & x_{2} & 0 & 0 & -x_{0} \\
-x_{1} & 0 & 0 & 0 & x_{3} & 0 & 0 \\
0 & -x_{2} & 0 & 0 & 0 & x_{4} & 0 \\
0 & 0 & -x_{3} & 0 & 0 & 0 & x_{5} \\
x_{6} & 0 & 0 & -x_{4} & 0 & 0 & 0 \\
0 & x_{0} & 0 & 0 & -x_{5} & 0 & 0
\end{array}\right) \\
\mu:=u_{0}^{t} u_{3}=u_{3}^{t} u_{0}=-u_{1}^{t} u_{1}= & \left(\begin{array}{ccccccc}
0 & 0 & 0 & -x_{5} & x_{2} & 0 & 0 \\
0 & 0 & 0 & 0 & -x_{6} & x_{3} & 0 \\
0 & 0 & 0 & 0 & 0 & -x_{0} & x_{4} \\
x_{5} & 0 & 0 & 0 & 0 & 0 & -x_{1} \\
-x_{2} & x_{6} & 0 & 0 & 0 & 0 & 0 \\
0 & -x_{3} & x_{0} & 0 & 0 & 0 & 0 \\
0 & 0 & -x_{4} & x_{1} & 0 & 0 & 0
\end{array}\right) \\
v:=u_{0}^{t} u_{1}=u_{1}^{t} u_{0}=-u_{2}^{t} u_{2}= & \left.\begin{array}{ccccccc}
0 & x_{4} & 0 & 0 & 0 & 0 & -x_{3} \\
-x_{4} & 0 & x_{5} & 0 & 0 & 0 & 0 \\
0 & -x_{5} & 0 & x_{6} & 0 & 0 & 0 \\
0 & 0 & -x_{6} & 0 & x_{0} & 0 & 0 \\
0 & 0 & 0 & -x_{0} & 0 & x_{1} & 0 \\
0 & 0 & 0 & 0 & -x_{1} & 0 & x_{2} \\
x_{3} & 0 & 0 & 0 & 0 & -x_{2} & 0
\end{array}\right)
\end{aligned}
$$

Furthermore $u_{0}^{t} u_{0}^{\#}=2 \cdot \operatorname{diag}\left(x_{0}, \ldots, x_{6}\right)$ and $u_{1}^{t} u_{0}^{\#}=v^{*}$, where $v^{*}$ is like $v$, but with all signs positive. Likewise $u_{2}^{t} u_{0}^{\#}=\lambda^{*}, u_{3}^{t} u_{0}^{\#}=\mu^{*}$. In the sequel we will omit to write $(\cdot)^{t}$, and we will identify loosely the elements of $\operatorname{Hom}\left(\Omega^{3}, \mathscr{O}(-3)\right)$ and $\operatorname{Hom}\left(\mathscr{O}(-4), \Omega^{3}\right)$.

In other words, once we choose a basis $u_{0}, \ldots, u_{3}$ of $\operatorname{Hom}_{G_{7}}\left(S \Omega^{3}, V_{4}\right.$ $\mathcal{O}(-3))$, we can view the matrix $\alpha$ associated to $A$ as an element of $M:=$ $M\left(3 \times 2, U^{\prime}\right)$. That is, the entries of this matrix are linear forms over $\mathrm{C}$ in four 
variables. We are going to give more information about this fact in the next sections.

Proposition 2.4 ([16], Proposition 3.5). A matrix $\alpha$ as in Theorem 2.1 satisfies $\alpha \alpha^{\prime}=0$ if and only if the three quadrics in $\mathrm{P}^{3}(U)$ given by its $2 \times 2$ minors (in $S^{2} U^{\prime}$ ) are annihilated by each of the three operators

$$
\Delta_{1}=2 a_{0} a_{1}-a_{2}^{2}, \quad \Delta_{2}=2 a_{0} a_{2}-a_{3}^{2}, \quad \Delta_{3}=2 a_{0} a_{3}-a_{1}^{2} .
$$

We denote by $\Delta$ the linear span of the operators $\Delta_{1}, \Delta_{2}, \Delta_{3}$, which is also the unique $\mathrm{SL}_{2}\left(\mathrm{Z}_{7}\right)$-invariant 3-dimensional subspace of $S^{2} U$. From Appendix A we see that $W \cong \Delta \subset S^{2} U$, so this property simply tells us that the minors of the matrix $\alpha$ lie in the irreducible $G_{7}$-module $L$, where $S^{2} U^{\prime}=L \oplus W^{\prime}$ (see Appendix A).

Remark 2.5. A resolution as in Theorem 2.1 is determined up to action from the left and right respectively of $\mathrm{GL}_{3}(\mathrm{C})$ and $\mathrm{GL}_{2}(\mathrm{C})$.

Let $C_{\alpha} \subset \mathrm{P}^{3}(U)$ be the curve defined by the $2 \times 2$ minors of a matrix $\alpha$ as in the resolution of Theorem 2.1.

Proposition 2.6 ([16], Proposition 4.1). The curve $C_{\alpha}$ is a projectively Cohen-Macaulay curve of degree 3 and arithmetic genus 0 .

This means that the minors of $\alpha$ determine a twisted cubic curve $C_{\alpha}$. In particular these minors are independent, so define a 3-dimensional linear subspace of $S^{2} U^{\prime}=H^{0}\left(\mathrm{P}^{3}(U), \mathscr{O}(2)\right)$. Furthermore it is shown in [16, Corollary 4.2] that if $A$ is smooth, then it is uniquely determined by the curve $C_{\alpha}$ associated to its resolution.

The Hilbert scheme $\operatorname{Hilb}_{3 t+1}\left(\mathrm{P}^{3}(U)\right)$ has two components of dimensions 12 and $15, \operatorname{Hilb}_{3 t+1}\left(\mathrm{P}^{3}(U)\right)=H_{1} \cup H_{2}$, with general points of $H_{1}, H_{2}$ and $H_{1} \cap H_{2}$ corresponding respectively to a twisted cubic, a plane cubic union a point and a plane nodal cubic with an embedded point at the node. If $C \in H_{1}$ then $\operatorname{dim} H^{0}\left(\mathrm{P}^{3}(U), \mathscr{I}_{C}(2)\right)=3$. The morphism

$$
\begin{aligned}
f: H_{1} & \longrightarrow \mathrm{G}\left(3, H^{0}\left(\mathrm{P}^{3}(U), \mathscr{O}(2)\right)\right) \\
C & \longmapsto H^{0}\left(\mathrm{P}^{3}(U), \mathscr{I}_{C}(2)\right)
\end{aligned}
$$

is birational onto its image $H$, and is an isomorphism precisely on $H_{1} \backslash\left(H_{1} \cap\right.$ $H_{2}$ ), cf. [5].

Consider

$$
H(\Delta):=H \cap \mathrm{G}(3, L) \subset \mathrm{G}\left(3, H^{0}\left(\mathrm{P}^{3}(U), \mathscr{O}(2)\right)\right),
$$


where $\mathrm{G}(3, L)$ is the Grassmannian of 3-dimensional vector subspaces of $L$. Since it does not intersect $f\left(H_{1} \cap H_{2}\right)$, we can regard $H(\Delta)$ as a subvariety of $H_{1}$ as well (cf. [16]).

TheOREM 2.7 ([16], Proposition 4.4). $H(\Delta)$ is a smooth prime Fano 3-fold of genus three.

By the results of [16] the moduli space of $(1,7)$-polarised abelian surfaces with canonical level structure is birational to $H(\Delta)$. For more information about Fano 3-folds, see [19], and about Fano 3-folds of genus 12, see [21].

\subsection{A different model of the moduli space}

We report a few crucial facts from [7] and [8]. The $7 \times 7$ matrix

$$
M_{7}^{\prime}(x, y)=\left(x_{\frac{i+j}{2}} y_{\frac{i-j}{2}}\right)_{i, j \in \mathrm{Z}_{7}}
$$

has rank at most 4 on an embedded $H_{7}$-invariant $(1,7)$-polarised abelian surface in $\mathrm{P}^{6}(V)$ : that is, if

$$
\left(\left(x_{0}: \ldots: x_{7}\right),\left(y_{0}: \ldots: y_{7}\right)\right) \in A \times A \subset \mathrm{P}^{6}(V) \times \mathrm{P}^{6}(V)
$$

then $\operatorname{rank} M \leq 4$ ([7, Corollary 2.8]).

On the other hand, for any parameter point $y=\left(0: y_{1}: y_{2}: y_{3}:-y_{3}\right.$ : $\left.-y_{2}:-y_{1}\right) \in \mathrm{P}^{2}\left(W^{\prime}\right)$, the matrix $M_{7}^{\prime}$ is alternating. We will denote in the sequel by $I_{3}(y) \subset \mathrm{C}\left[x_{0}, \ldots, x_{6}\right]$ the homogeneous ideal generated by the $6 \times 6$ Pfaffians of the alternating matrix $M_{7}^{\prime}(x, y)$ and by $V_{7, y} \subset \mathrm{P}^{6}(V)$ the closed subscheme defined by this ideal. Notice that, in our notation, $M_{7}^{\prime}(x,(1$ : $0: 0))=\lambda, M_{7}^{\prime}(x,(0: 1: 0))=\mu$ and $M_{7}^{\prime}(x,(0: 0: 1))=v$.

Now we quote two propositions of Gross and Popescu that we are going to need.

Proposition 2.8 ([7], Proposition 5.2). Let $y \in \mathrm{P}^{2}\left(W^{\prime}\right)$.

(1) For $y \in Q^{\prime}=\left\{y_{1}^{3} y_{2}+y_{2}^{3} y_{3}+y_{3}^{3} y_{1}=0\right\} \subset \mathrm{P}^{2}\left(W^{\prime}\right)$, the scheme $V_{7, y}$ is the secant variety of an elliptic normal curve in $\mathrm{P}^{6}(V)$ (the level 7 elliptic curve corresponding to the point $y$ on the modular curve $Q^{\prime}$ ).

(2) For a general $y \in \mathrm{P}^{2}\left(W^{\prime}\right)$, the scheme $V_{7, y}$ is a projectively Gorenstein irreducible threefold of degree 14 and sectional genus 15.

Proposition 2.9 ([7], Proposition 5.4). Let $A \in \mathrm{P}^{6}(V)$ be a general Heisenberg-invariant $(1,7)$-polarised abelian surface, and let $A \cap \mathrm{P}^{2}\left(W^{\prime}\right)=$ $\left\{p_{1}, \ldots, p_{6}\right\}$ be the odd 2-torsion points of $A$. Then:

(1) The points $p_{i}$ form a polar hexagon to the Klein quartic curve $Q \subset$ $\mathrm{P}^{2}(W)$. 
(2) The surface $A$ is contained in $V_{7, p_{i}}$, for all $i=1, \ldots, 6$. Moreover, 21 cubic Pfaffians defining any three of the six $V_{7, p_{i}}$ generate the homogeneous ideal $I_{A}$ of $A$.

It follows that the moduli space of $(1,7)$-polarised abelian surfaces with canonical level structure is birational to the space $\operatorname{VSP}(Q, 6)$ of polar hexagons to the Klein quartic curve $Q \subset \mathrm{P}^{2}(W)$.

Remark 2.10. By direct computation we see that the seven Pfaffians of a matrix $M_{7}^{\prime}(x, y)$ associated to an element $y=\left(0: y_{1}: y_{2}: y_{3}:-y_{3}:-y_{2}\right.$ : $\left.-y_{1}\right) \in \mathrm{P}^{2}\left(W^{\prime}\right)$ are given by $\left.y^{3}\right|_{L} \otimes V_{4}$, where from the appendix we have $S^{3} W^{\prime}=L \oplus W$ and $S^{3} V_{3}=\left(I \oplus U^{\prime} \oplus L\right) \otimes V_{4}$ : i.e. the $i$-th Pfaffian is

$$
\begin{aligned}
& \operatorname{Pfaff}_{i}\left(y_{1} \lambda+y_{2} \mu+y_{3} \nu\right) \\
& =\left(y_{2}^{2} y_{3}-y_{1}^{3}\right) x_{i} x_{i+3} x_{i+4}+\left(y_{3}^{2} y_{1}-y_{2}^{3}\right) x_{i} x_{i+1} x_{i+6}+\left(y_{1}^{2} y_{2}-y_{3}^{3}\right) x_{i} x_{i+2} x_{i+5} \\
& +y_{1} y_{2} y_{3}\left(x_{i+1} x_{i+2} x_{i+4}+x_{i+3} x_{i+5} x_{i+6}-x_{i}^{3}\right)+y_{1}^{2} y_{3}\left(x_{i+2}^{2} x_{i+3}+x_{i+5}^{2} x_{i+4}\right) \\
& +y_{2}^{2} y_{1}\left(x_{i+3}^{2} x_{i+1}+x_{i+4}^{2} x_{i+6}\right)+y_{3}^{2} y_{2}\left(x_{i+1}^{2} x_{i+5}+x_{i+6}^{2} x_{i+1}\right) .
\end{aligned}
$$

Then Proposition 2.9(ii) simply says that the linear space spanned by any three of the $\left.\operatorname{six} p_{i}^{3}\right|_{L} \in L$ is 3-dimensional.

\section{The geometry of $H(\Delta)$ and its boundary}

\subsection{The moduli space as an orbit space}

In what follows we regard the moduli space of $(1,7)$-polarised abelian varieties as an orbit space: in fact, by Remark $2.5, \mathrm{GL}_{3}(\mathrm{C})$ and $\mathrm{GL}_{2}(\mathrm{C})$ act on the variety $M:=M\left(3 \times 2, U^{\prime}\right)$. In order to apply the theory of orbit spaces, see [20], p. 73, we restrict our attention to $\mathrm{P}(M), \mathrm{SL}_{3}(\mathrm{C})$ and $\mathrm{SL}_{2}(\mathrm{C})$. Doing so, no harm has been done to the elements

$$
H^{0}\left(\mathrm{P}^{3}(U), \mathscr{I}_{\alpha}(2)\right) \in \mathrm{G}\left(3, H^{0}\left(\mathrm{P}^{3}(U), \mathcal{O}(2)\right)\right),
$$

where $\mathscr{I}_{\alpha}$ is the ideal generated by the minors $q_{i}(\alpha)$ of a matrix $\alpha$ arising from a resolution as in Subsection 2.3, and to the effects of the actions.

Notice that the actions of $\mathrm{SL}_{3}(\mathrm{C})$ and $\mathrm{SL}_{2}(\mathrm{C})$ on $\mathrm{P}(M)$ induce actions on the minors, given by linear combinations. In any case the vector space $W_{\alpha}$ generated by the minors of $\alpha$ is fixed by this action. Notice that $\mathrm{SL}_{3}(\mathrm{C}) \times \mathrm{SL}_{2}(\mathrm{C})$ is reductive, and we consider its obvious linearisation.

The study of this problem has been done in [5] and gives the following result:

Proposition 3.1. Let $\alpha \in M$. The following are equivalent: 
(1) $\alpha$ is stable,

(2) $\alpha$ is semi-stable,

(3) the minors of $\alpha$ are linearly independent.

Now let $\mathrm{P}(M)^{s}$ be the set of the stable points of $\mathrm{P}(M) . \mathrm{P}(M)^{s}$ is open, so quasi-projective (cf. [20]). Let $\mathrm{P}(M)_{\Delta}^{s}$ be the subvariety of $\mathrm{P}(M)^{s}$ defined by the nine quadratic conditions (three for each minor) $\Delta_{i}\left(q_{j}(\alpha)\right)=0$.

Proposition 3.2. There is a projective variety $Y$ and an affine surjective morphism $\phi$ such that $\phi: \mathrm{P}(M)^{s} \rightarrow Y$ is a geometric quotient and $\phi\left(\mathrm{P}(M)_{\Delta}^{s}\right):=\mathscr{Y}$ is projective as well. Furthermore distinct orbits are mapped to distinct elements of $Y$ (and so of $\mathscr{Y}$ as well).

Proof. After noting that a stable orbit of $\mathrm{P}(M)$ is either entirely in $\mathrm{P}(M)_{\Delta}^{s}$ or entirely outside, everything follows from [20], Theorem 3.14 and Theorem 3.5(iv).

LeMma 3.3. Let $\alpha_{1}, \alpha_{2} \in M\left(3 \times 2, U^{\prime}\right)$ be two matrices whose minors span a 3-dimensional subspace. If $\left\langle q_{i}\left(\alpha_{1}\right)\right\rangle_{i=1,2,3}=\left\langle q_{i}\left(\alpha_{2}\right)\right\rangle_{i=1,2,3}$, then $\alpha_{1}$ and $\alpha_{2}$ lie in a common orbit under the action of $\mathrm{GL}_{3}(\mathrm{C}) \times \mathrm{GL}_{2}(\mathrm{C})$.

Proof. First of all, up to the action, we can rearrange the matrices in such a way that $q_{i}\left(\alpha_{1}\right)=q_{i}\left(\alpha_{2}\right):=q_{i}, i=1,2,3$. Obviously the ideals these minors generate define the same variety $Z \in \mathrm{P}^{3}(U)$. The obvious resolution

$$
0 \longrightarrow 2 \mathscr{O}(-3) \stackrel{\alpha_{1}}{\longrightarrow} 3 \mathscr{O}(-2) \stackrel{\left(q_{i}\right)}{\longrightarrow} \mathscr{O} \longrightarrow \mathscr{O}_{Z} \longrightarrow 0
$$

is unique up to action by $\mathrm{GL}_{2}(\mathrm{C})$, and it still works if we replace $\alpha_{1}$ with $\alpha_{2}$. The columns of $\alpha_{1}$ and $\alpha_{2}$ are just the syzygies of $q_{i}, i=1,2,3$ (see Theorem 5.2), thus there is some $x \in \mathrm{GL}_{2}(\mathrm{C})$ such that $\alpha_{1}=\alpha_{2} x$.

This lemma allows us to prove the main result of this subsection:

Proposition 3.4. Y is isomorphic to $H(\Delta)$.

Proof. Consider the morphism $\omega: \mathrm{P}(M)_{\Delta}^{s} \rightarrow H(\Delta)$ given by $\alpha \mapsto$ $W_{\alpha}$. Obviously $\omega$ is constant on orbits, and since $\mathrm{G}(3, L)$ does not intersect $f\left(H_{1} \cap H_{2}\right)$, any of its elements is representable by some element of $\mathrm{P}(M)_{\Delta}^{s}$, and then $\omega$ is surjective as well. By [20, Corollary 3.5.1], we see that $(\mathscr{Y}, \phi)$ is a categorical quotient of $\mathrm{P}(M)_{\Delta}^{s}$. By definition of categorical quotient (see [20], definition after Proposition 2.9), there is a (unique) morphism $\psi: \mathscr{Y} \rightarrow H(\Delta)$ such that $\omega=\psi \circ \phi$.

By Lemma 3.3 and Proposition 3.2, $\psi$ is a bijective morphism, and by [19] $H(\Delta)$ is a smooth (normal) irreducible variety. Consider the normalisation $v: \tilde{\mathscr{Y}} \rightarrow \mathscr{Y}$. In particular $\psi \circ v$ is a normalisation of $H(\Delta)$, so by [22] (corollary 
to Theorem 5 in Section 2) we can conclude that $\psi \circ v$ is the identity and then $\psi$ is actually an isomorphism.

\subsection{Geometry of the boundary $B$}

In this subsection we study the geometry of the boundary $B$ of the moduli space of twisted cubic curves annihilated by $\Delta$. The key observation is that by [9], Proposition 9.4, if a matrix $\alpha \in M$ is not conjugate to a matrix $\alpha^{\prime}$ having a 0 -entry, then the minors of $\alpha$ determine a twisted cubic curve. Obviously, if the minors of $\alpha \in M$ define a twisted cubic curve, it cannot be conjugate to a matrix having a 0 -entry. This means that in this subsection we are studying the subset $B$ of $H(\Delta)$ defined by the condition that its elements are the images via $\phi$ of the set of all the matrices conjugate to some matrix having a 0 -entry.

Lemma 3.5. $B$ is a closed subvariety of $H(\Delta)$.

Proof. Consider the subvariety $\hat{B}$ of $\mathrm{P}(M)_{\Delta}^{s} \times \mathrm{G}(1,2) \times \mathrm{G}(2,3) \cong \mathrm{P}(M)_{\Delta}^{s}$ $\times \mathrm{P}^{1} \times \mathrm{P}^{2}$ defined by the condition that $(\alpha, a, b) \in \hat{B}$ if and only if $\alpha_{i}(a) \subset b$, $i=0, \ldots, 3$, where $\alpha_{i}$ is the matrix given by projecting the entries of $\alpha$ to $\left\langle u_{i}\right\rangle$. In other words, $(\alpha, a, b) \in \hat{B}$ if $a$ is a 1-dimensional subspace of $\mathrm{C}^{2}$ whose image is contained in the 2-dimensional subspace $b$ of $\mathrm{C}^{3}$ for every $\alpha_{i}$; and this is the case if and only if $\alpha$ is conjugate to a matrix of $M$ having a 0 -entry. Clearly $\hat{B}$ is closed, and so is its projection $\left.\pi\right|_{M_{\Delta}^{s}}(\hat{B})$ into $\mathrm{P}(M)_{\Delta}^{s}$, see [9], Theorem 3.12. Moreover it is invariant under the action of $\mathrm{SL}_{3}(C) \times \mathrm{SL}_{2}(C)$. Finally, by [20, Theorem 3.5.iv], we get that $\phi\left(\left.\pi\right|_{\mathrm{P}(M)_{\Delta}^{s}}(\hat{B})\right)=B$ is closed in $H(\Delta)$.

The next step is to find a suitable representative for each $[\alpha] \in B$. In other words, we want to parametrise the boundary $B$ of $H(\Delta)$. We can suppose that $\alpha_{12}=0$. For simplicity from now on we write

$$
\alpha=\left(\begin{array}{ll}
a & 0 \\
b & d \\
c & e
\end{array}\right) .
$$

If we write $a=\sum_{i=0}^{3} a_{i} u_{i}$, etc., then the minors $a d$ and $a e$ satisfy $\Delta$ if and only if $d$ and $e$ satisfy the linear system

$$
a^{*}=\left(\begin{array}{cccc}
a_{1} & a_{0} & -a_{2} & 0 \\
a_{2} & 0 & a_{0} & -a_{3} \\
a_{3} & -a_{1} & 0 & a_{0}
\end{array}\right)
$$

REMARK 3.6. Let $x$ and $y$ be any two elements of $U^{\prime}$. Via the previous matrix $x^{*}$ we get a multiplication $U^{\prime} \times U^{\prime} \rightarrow W^{\prime} \subset S^{2} U^{\prime}=L \oplus W^{\prime}$ given 
by $(x, y) \mapsto x^{*} y$. Notice that these are all $\mathrm{SL}_{2}\left(\mathrm{Z}_{7}\right)$-modules. This induces a product $*: U^{\prime} \times U^{\prime} \rightarrow W^{\prime}$. Obviously $*$ is symmetric, and the condition $\Delta$ says that every minor of a matrix $\alpha$ is contained in $L \subset S^{2} U^{\prime}$. This is a fact we are going to use heavily in the next section.

The rank of $a^{*}$ must be at most 2, because otherwise $d$ and $e$ would be dependent, and so a minor of $\alpha$ would be zero. Thus the minors of $a^{*}$ must vanish, that is

$$
\begin{aligned}
a_{0}^{3}-a_{1} a_{2} a_{3} & =0 \\
a_{0}^{2} a_{1}+a_{0} a_{2}^{2}+a_{2} a_{3}^{2} & =0 \\
a_{0}^{2} a_{2}+a_{0} a_{3}^{2}+a_{3} a_{1}^{2} & =0 \\
a_{0}^{2} a_{3}+a_{0} a_{1}^{2}+a_{1} a_{2}^{2} & =0
\end{aligned}
$$

Let us call $\Gamma$ the curve in $\mathrm{P}^{3}\left(U^{\prime}\right)$ defined by these equations. As shown in Appendix B, $\Gamma$ is isomorphic to the modular curve of level 7, whose standard model is the Klein quartic $Q$ given by the equation (21).

It is easy to check that the rank of $a^{*}$ cannot be 1 . So the space of solutions is 2-dimensional, and then we can fix a basis for it, namely $(d, e)$. In other words, as long as $d$ and $e$ are independent, any choice we make is good, and does not affect the space spanned by the minors of the matrix.

Lemma 3.7. Let $\alpha \in M^{s}$. Then none of its minors is of the form $l^{2}$, where $l$ is a linear form.

Proof. Suppose $l=l_{0} u_{0}+l_{1} u_{1}+l_{2} u_{2}+l_{3} u_{3}$ and $l^{2}$ satisfies $\Delta$. Then we can certainly suppose that $l_{0}=1$, and from $\alpha^{*}$ (with $l$ in place of $a$ ), we get $2 l_{i}=l_{i+1}^{2}, i=1,2,3$. Thus, for example, $l_{2}=2 \xi$, where $\xi^{7}=1$, but then $l_{1} l_{2} l_{3}=2 \xi 2 \xi^{2} 2 \xi^{4}=8 \neq 1$, so the linear system $a^{*}$ would have rank 3 , contradicting the fact that $\alpha \in M^{s}$.

This lemma implies that, because of the $\Delta$ condition, the variety defined by the minors of $\alpha$ is a (possibly reducible) curve: contrast [5, Lemma 3].

Proposition 3.8. $B$ is birational to $\Gamma \times \mathrm{P}^{1} \cong Q \times \mathrm{P}^{1}$.

Proof. If, as in (3), $\alpha$ is a matrix representing the point $[\alpha] \in B$, then $a \in \Gamma$, while $d$ and $e$ are determined up to choice of a basis. We call $C_{\alpha}$ the variety defined by the minors of $\alpha$.

For the minor $q_{1}(\alpha)$ to satisfy $\Delta$, the vector $(b, c)$ must satisfy the linear 
system

$$
\Lambda=\left(\begin{array}{cccccccc}
e_{1} & e_{0} & -e_{2} & 0 & -d_{1} & -d_{0} & d_{2} & 0 \\
e_{2} & 0 & e_{0} & -e_{3} & -d_{2} & 0 & -d_{0} & d_{3} \\
e_{3} & -e_{1} & 0 & e_{0} & -d_{3} & d_{1} & 0 & -d_{0}
\end{array}\right) .
$$

If $a_{0}=0$, by (4), ., (7) two more coefficients among $a_{1}, a_{2}$ and $a_{3}$ must be zero. We can suppose $a_{1}=1$, and so $d, e \in\left\langle u_{2}, u_{3}\right\rangle$. Let $d=u_{2}$ and $e=u_{3}$. Then we can suppose $b_{1}=b_{2}=c_{1}=0$, so $b_{3}=-c_{0}, b_{0}=c_{2}=0$ and $c_{3}$ is arbitrary. Therefore

$$
\alpha=\left(\begin{array}{cc}
u_{1} & 0 \\
b_{3} u_{3} & u_{2} \\
-b_{3} u_{0}+c_{3} u_{3} & u_{3}
\end{array}\right)
$$

These matrices are parametrised by $\left(b_{3}: c_{3}\right) \in \mathrm{P}^{1}$. Unless $b_{3}=0$, their minors determine a line and a conic, non-coplanar and meeting at a point. If $b_{3}=0$ the minors determine three non-coplanar lines meeting at a common point.

If $a_{0} \neq 0$ we can set $a_{0}=1$, and (4) gives us $a_{3}=1 / a_{1} a_{2}$, while (5), (6) and (7) become the non-homogeneous equation $1+a_{1}^{2} a_{2}^{3}+a_{1}^{3} a_{2}=0$. It is easy to see that either $d_{0}$ or $e_{0}$ is nonzero, so let $e_{0}=1$, and $d_{0}=0$, which implies $d_{1} d_{2} d_{3} \neq 0$. We can assume that $b_{0}=b_{1}=c_{0}=0$, so now the vector $\left(b_{2}, b_{3}, c_{1}, c_{2}, c_{3}\right)$ must satisfy the linear system

$$
\Lambda^{\prime}=\left(\begin{array}{ccccc}
-e_{2} & 0 & 0 & d_{2} & 0 \\
1 & -e_{3} & 0 & 0 & d_{3} \\
0 & 1 & d_{1} & 0 & 0
\end{array}\right)
$$

The rank of $\Lambda^{\prime}$ is 3, so we get a 2-dimensional space of solutions. Obviously multiplication by a scalar on $(b, c)$ does not affect $C_{\alpha}$, and so we get a $\mathrm{P}^{1}$ of solutions. Also in this case in general $C_{\alpha}$ is a conic and a line, non-coplanar and meeting at a point. If this does not happen, then $C_{\alpha}$ can only be the union of three non-coplanar lines, one of them meeting the other two, possibly at a single point. If so, by Lemma 3.3 the minors of $\alpha$ can be arranged in such a way that

$$
\alpha=\left(\begin{array}{ll}
a & 0 \\
b & d \\
0 & e^{\prime}
\end{array}\right)
$$

So we are looking for a form $e^{\prime}=x d+y e \in \Gamma$. Actually $e^{\prime}=x d+e$, because 
$d \notin \Gamma$. Consider the linear system

$$
\left(\begin{array}{cccc}
e_{1}^{\prime} & 1 & -e_{2}^{\prime} & 0 \\
e_{2}^{\prime} & 0 & 1 & -e_{3}^{\prime} \\
e_{3}^{\prime} & -e_{1}^{\prime} & 0 & 1
\end{array}\right)
$$

Obviously $a$ satisfies it, and since we can assume that $b_{0}=0$, the minor

$$
1-\left(e_{1}+x d_{1}\right)\left(e_{2}+x d_{2}\right)\left(e_{3}+x d_{3}\right)
$$

must be zero.

REMARK 3.9. If $e^{\prime} \in\langle d, e\rangle$ satisfies (9), then as this implies $e^{\prime} \in \Gamma$, we get that $e^{\prime}$ satisfies $(4), \ldots,(7)$.

From $d_{1} d_{2} d_{3} \neq 0$ we conclude that the degree of the previous polynomial is 3 , and so for any given $a$ (with $a_{0} \neq 0$ ) we get in general three matrices (and then elements of $B$ ) whose minors define three lines.

So far we have proved that over every point $a \in \Gamma$ there is a $\mathrm{P}^{1}$ of elements of $B$. That is, the closures of the fibres of the rational map $B \rightarrow \Gamma \cong Q$ given by $\alpha \mapsto a$ are $\mathrm{P}^{1}$ 's. The map is not well-defined if and only if an element of $B$ is represented by a matrix like (8). Indeed $a$ determines the plane where the conic lives, and so if the conic is smooth, $a$ must be unique.

Therefore now we only need to show that the locus $B^{\prime} \subset B$ whose elements are represented by matrices like $(8)$ is closed. This is clear after noticing that

$$
B^{*}:=\left\{(a, b) \in \Gamma \times \Gamma \mid a^{*} b=0\right\} \subset \Gamma \times \Gamma \subset \mathrm{P}^{3}\left(U^{\prime}\right) \times \mathrm{P}^{3}\left(U^{\prime}\right)
$$

is a proper closed subset of $\Gamma \times \Gamma$, and that $B^{\prime}$ is isomorphic to $B^{*}$, in fact the entries $b$ (respectively $d^{\prime}$ ) of (8) can be chosen arbitrarily among the solutions of $e^{*}$ (respectively $a^{*}$ ), and we are still in the same orbit of matrices representing $\xi \in B^{\prime}$. Notice that the projections $p_{i}: B^{*} \rightarrow \Gamma, i=1,2$ are generically $3: 1$.

From (9) we see that in general a fibre of $B$ over a point $a \in \Gamma$ meets three other fibres, each one at a single point. In the next lemma we work out over which fibres the intersection is non-smooth.

Lemma 3.10. Let $\alpha$ be a matrix whose image is in B, and let the top-left entry be $a \in \Gamma$. If the rational fibre over a does not meet three distinct fibres, then $a$ is the image of a cusp of $Q$.

Proof. The first observation is that over the cusp $a=u_{1}$, corresponding to $y_{2}$, where $y_{i}$ is the dual basis of $v_{i}$, the space of solutions of $a^{*}$ is given by the last two columns of (27) (see Appendix B), that is $\left\langle u_{2}, u_{3}\right\rangle$. Then after 
replacing the polynomial (9) with the polynomial given by (5), we get clearly a double solution, namely $e_{2}^{\prime} e_{3}^{\prime 2}=0$.

Now notice that, by Remark 3.9, the condition that the polynomials like (9) have a double solution is invariant under the action of $\mathrm{SL}_{2}\left(\mathrm{Z}_{7}\right)$, whose action on the entries (in $U^{\prime}$ ) of $\alpha$ descends to an action on the coefficients of the polynomials. Therefore the set of points of $\Gamma$ we are after is a union of $\mathrm{SL}_{2}\left(\mathrm{Z}_{7}\right)$-orbits of $\Gamma$, and it contains the orbit of 24 images of cusps of $Q$.

Let us assume $a_{0} \neq 0$. Then we only need the polynomial (9) to have a double solution. Using as $d$ and $e$ the first two columns of (27), the discriminant $D$ of (9), divided by a common monomial, has degree 11 , and is not divisible by $f_{\text {Klein }}$. Then the zero locus of $D$ intersects $Q$ at at most 44 reduced points, including the three cusps with zero entries. By [13], there is one orbit only with at most 44 points, precisely the orbit of 24 cusps.

Remark 3.11. As mentioned in the summary, at first sight we have a good candidate for a possible subspace of $H(\Delta)$ whose points could parametrise translation scrolls. In fact we have just seen in Proposition 3.8 that $B$ is birational to the Kummer modular surface that parametrises those surfaces. But things will not be as straightforward as expected, as we will see in Section 5. For a brief description of the translation scrolls, see Section 4, where everything works for $(1, p)$ in place of $(1,5)$.

\subsection{More on the isomorphic models of the moduli space}

We have seen that the moduli space we are interested in is birational to $H(\Delta)$, the moduli space of twisted cubic curves annihilated by the net of quadrics $\Delta$ and also to the variety of sum of powers $\operatorname{VSP}(Q, 6)$, where

$\operatorname{VSP}(Q, 6)$

$$
=\overline{\left\{\left\{l_{1} \ldots l_{6}\right\} \in \operatorname{Hilb}_{6}\left(\mathrm{P}^{2}\left(W^{\prime}\right)\right) \mid l_{1}^{4}+\ldots+l_{6}^{4}=v_{1}^{3} v_{2}+v_{2}^{3} v_{3}+v_{3}^{3} v_{1}\right\}} .
$$

In fact $H(\Delta)$ and $\operatorname{VSP}(Q, 6)$ are isomorphic, and in this subsection we are going to discuss the isomorphism in more detail and present more facts on these spaces.

Theorem 3.12 ([21], Theorem 1.1). There are isomorphisms

$$
H(\Delta) \stackrel{\sim}{\longrightarrow} \mathrm{G}\left(3, L, \eta_{\text {Klein }}\right) \stackrel{\sim}{\longrightarrow} \operatorname{VSP}(Q, 6) .
$$

As explained in [21], every Fano 3-fold of genus 12 has these descriptions over an algebraically closed field of characteristic zero. $\mathrm{G}\left(3, L, \eta_{\text {Klein }}\right)$ 
is as follows (notice that the spaces here correspond to those in Appendix A): consider on

$L=\left(f_{\text {Klein }}^{\perp}\right)_{3}^{*}=\left\langle v_{1} v_{2} v_{3}, v_{2} v_{3}^{2}, v_{3} v_{1}^{2}, v_{1} v_{2}^{2}, v_{3}^{2} v_{1}-v_{2}^{3}, v_{1}^{2} v_{2}-v_{3}^{3}, v_{2}^{2} v_{3}-v_{1}^{3}\right\rangle$ the net $\eta_{\text {Klein }}: \wedge^{2} L \rightarrow W \cong \mathrm{C}^{3}$ of alternating forms defined by the matrix

$$
\eta_{\text {Klein }}=\left(\begin{array}{rrrrrrr}
0 & 0 & 0 & 0 & 0 & -y_{2} & y_{1} \\
0 & 0 & 0 & 0 & -y_{3} & 0 & y_{2} \\
0 & 0 & 0 & -y_{1} & 0 & 0 & y_{3} \\
0 & 0 & y_{1} & 0 & y_{2} & -y_{3} & 0 \\
0 & y_{3} & 0 & -y_{2} & 0 & y_{1} & 0 \\
y_{2} & 0 & 0 & y_{3} & -y_{1} & 0 & 0 \\
-y_{1} & -y_{2} & -y_{3} & 0 & 0 & 0 & 0
\end{array}\right) .
$$

Here $\left(f_{\text {Klein }}^{\perp}\right)_{3}=\left\{x \in S^{3} W=\mathrm{C}\left[y_{1}, y_{2}, y_{3}\right]_{3} \mid x f_{\text {Klein }}=0\right\}$, the 7-dimensional vector space of differentials of the third order that annihilates $f_{\text {Klein }}$ (see [21]). We write $y_{i}$ in place of $\partial v_{i}$ for the dual generators of $W$ with respect to the $v_{i}$, the elements of the basis of $W^{\prime}$ in the appendix. Then

$$
\mathrm{G}\left(3, L, \eta_{\text {Klein }}\right)=\left\{E \in \mathrm{G}(3, L) \mid \wedge^{2} E \subset \operatorname{ker}\left(\eta_{\text {Klein }}: \wedge^{2} L \rightarrow W\right)\right\} .
$$

We follow [21, Theorem 2.6] to give a sketch of the proof of the second isomorphism in (10).

The Pfaffians of $\eta_{\text {Klein }}$ are $\left(f_{\mathrm{Klein}}^{\perp}\right)_{3}$, and if $I_{\text {Pfaff }}$ is the ideal they generate, then the dual socle generator (see [4]) of C $\left[y_{1}, y_{2}, y_{3}\right] / I_{\text {Pfaff }}$ is $f_{\text {Klein }}$.

Given an element $P \in \mathrm{G}\left(3, L, \eta_{\text {Klein }}\right)$, choose a basis $l$ for $L$ with the last three generators being taken from a basis $p$ for $P$. Then, with respect to this chosen basis, $\eta_{\text {Klein }}$ gets a block decomposition form

$$
\eta_{\text {Klein }} \sim \eta_{\text {Klein }}^{\prime}=\left(\begin{array}{cc}
* & \psi \\
-\psi^{t} & 0
\end{array}\right)
$$

Now $\psi$ can be viewed as a $4 \times 3$ syzygy matrix for the exact complex

$$
0 \longrightarrow 3 \mathcal{O}_{\mathrm{P}^{2}\left(W^{\prime}\right)}(-4) \stackrel{\psi}{\longrightarrow} 4 \mathcal{O}_{\mathrm{P}^{2}\left(W^{\prime}\right)}(-3) \stackrel{\text { minors }}{\longrightarrow} \mathcal{O}_{\mathrm{P}^{2}\left(W^{\prime}\right)} \longrightarrow \mathscr{O}_{\Xi} \longrightarrow 0,
$$

where $\Xi \subset \mathrm{P}^{2}\left(W^{\prime}\right)$ is six points, simply by computing the Hilbert series: $P_{\text {Hilb }}(\Xi)=(1,3,6,6,6 \ldots)$.

Notice that if we replace $p$ with $p^{\prime}=p a, a \in \mathrm{GL}_{3}$, then we simply get $\psi^{\prime}=\psi a$, and the above resolution is not affected. Similarly if we choose differently the first four generators of $l$, then only the top-left block $*$ of $\eta_{\text {Klein }}^{\prime}$ will be affected. 
Consider the first four Pfaffians of $\eta_{\text {Klein }}^{\prime}$. They must be a linear combination of the original ones, and are given by the minors of $\psi$, so we see that $\left(I_{\Xi}\right)_{3} \subset$ $\left(f_{\text {Klein }}^{\perp}\right)_{3}$. Thus

$$
S^{3} W \rightarrow R_{\Xi}=\mathrm{C}\left[y_{1}, y_{2}, y_{3}\right] / I_{\Xi} \rightarrow R_{\text {Klein }}=\mathrm{C}\left[y_{1}, y_{2}, y_{3}\right] /\left(f_{\text {Klein }}^{\perp}\right)
$$

and

$$
\operatorname{Hom}\left(\left(R_{\text {Klein }}\right)_{4}, \mathrm{C}\right) \subset \operatorname{Hom}\left(\left(R_{\Xi}\right)_{4}, \mathrm{C}\right) .
$$

Finally, $f_{\text {Klein }}$ is a generator for $\operatorname{Hom}\left(\left(R_{\text {Klein }}\right)_{4}, \mathrm{C}\right)$, being the dual socle generator, and the fourth power of the six points $\gamma_{i}$ of $\Xi \subset W^{\prime}$ impose independent conditions on quartics. Then $\left\langle\gamma_{i}^{4}\right\rangle_{i=1, \ldots, 6}=\operatorname{Hom}\left(\left(R_{\Xi}\right)_{4}, \mathrm{C}\right)$, so

$$
f_{\text {Klein }}=\sum_{i=1}^{6} \lambda_{i} \gamma_{i}^{4} .
$$

The first isomorphism in (10) comes from [21, Theorem 5.1], and in our picture it is clear after we notice that, with the notation from the appendix, the correspondence with the data in [21] is given by the net of quadrics $q(W)=$ $\Delta \subset S^{2} U$ and by the 7-dimensional space annihilated by $\Delta$,

$$
\begin{aligned}
\left(\Delta^{\perp}\right)_{2} & =\left\langle u_{0}^{2}, u_{2} u_{3}, u_{3} u_{1}, u_{1} u_{2}, u_{0} u_{3}+u_{1}^{2}, u_{0} u_{1}+u_{2}^{2}, u_{0} u_{2}+u_{3}^{2}\right\rangle \\
& =L \subset S^{2} U^{\prime}=L \oplus W^{\prime} .
\end{aligned}
$$

Then (see $[21, \S 5]) \eta_{q}=\eta_{\text {Klein }}$, where $\eta_{q}$ is a skew-symmetric matrix one can recover from the resolution of the module $S U^{\prime} /\left(\Delta^{\perp}\right)$. Let $H(q)$ denote the variety of twisted cubics $C \subset \mathrm{P}^{3}(U)$ whose equations $H^{0}\left(\mathrm{P}^{3}(U), I_{C}(2)\right) \subset$ $S^{2} U^{\prime}$ are annihilated by $q$. Since a twisted cubic is defined by the quadrics that contain it and $h^{0}\left(\mathrm{P}^{3}(U), I_{C}(2)\right)=3, H(q)$ is a subset of $\mathrm{G}\left(3, V_{q}\right)$ in a natural way. Then one can prove (see [21]) that $\eta_{q}$ is a net of alternating forms on $V_{q}$ which defines $H(q) \subset \mathrm{G}\left(3, V_{q}\right)$, namely

$$
H(\Delta) \cong \mathrm{G}\left(3, V_{q}, \eta_{q}\right)=\mathrm{G}\left(3, V_{\Delta}, \eta_{\text {Klein }}\right) .
$$

We make use of the fact that $L$ is self dual: the copies of $L$ we use are $\left(f_{\text {Klein }}^{\perp}\right)_{3}^{*} \subset$ $S^{3} W^{\prime}=L \oplus W$ and $V_{\Delta} \subset S^{2} U^{\prime}=L \oplus W^{\prime}$.

We can describe the $\mathrm{SL}_{2}\left(\mathrm{Z}_{7}\right)$-isomorphism $S^{3} W \stackrel{\sim}{\rightarrow} L \oplus W^{\prime}=S^{2} U^{\prime}$ by identifying

(12) $y_{1} y_{2} y_{3}=u_{0}^{2}, \quad y_{2} y_{3}^{2}=u_{2} u_{3}, \quad y_{3} y_{1}^{2}=u_{3} u_{1}, \quad y_{1} y_{2}^{2}=u_{1} u_{2}$, $y_{3}^{2} y_{1}-y_{2}^{3}=u_{0} u_{3}+u_{1}^{2}, \quad y_{1}^{2} y_{2}-y_{3}^{3}=u_{0} u_{1}+u_{2}^{2}, \quad y_{2}^{2} y_{3}-y_{1}^{3}=u_{0} u_{2}+u_{3}^{2}$. 
Similarly we can describe the dual isomorphism by

$$
a_{2} a_{3}=v_{2} v_{3}^{2}, \quad a_{1} a_{2}=v_{1} v_{2}^{2}, \quad a_{3} a_{1}=v_{3} v_{1}^{2}, \quad a_{0}^{2}=v_{1} v_{2} v_{3},
$$

$2 a_{0} a_{1}+a_{2}^{2}=v_{1}^{2} v_{2}-v_{3}^{3}, \quad 2 a_{0} a_{2}+a_{3}^{2}=v_{2}^{2} v_{3}-v_{1}^{3}, \quad 2 a_{0} a_{3}+a_{1}^{2}=v_{3}^{2} v_{1}-v_{2}^{3}$,

where the $a_{i}$ 's are the duals of the $u_{i}$ 's.

The $\mathrm{SL}_{2}\left(Z_{7}\right)$-isomorphism between the above copies of $W^{\prime}$ is given by

$3 y_{3}^{2} y_{1}+y_{2}^{3}=u_{0} u_{3}-u_{1}^{2}, \quad 3 y_{1}^{2} y_{2}+y_{3}^{3}=u_{0} u_{1}-u_{2}^{2}, \quad 3 y_{2}^{2} y_{3}+y_{1}^{3}=u_{0} u_{2}-u_{3}^{2}$,

and this leads to two isomorphic representations of $W$ given by

$3 v_{3}^{2} v_{1}+v_{2}^{3}=2 a_{0} a_{3}-a_{1}^{2}, \quad 3 v_{1}^{2} v_{2}+v_{3}^{3}=2 a_{0} a_{1}-a_{2}^{2}, \quad 3 v_{2}^{2} v_{3}+v_{1}^{3}=2 a_{0} a_{2}-a_{3}^{2}$.

This allows us to map the generators of a twisted cubic curve, namely the minors of a $3 \times 2$ matrix with linear entries in $U^{\prime}$, which are annihilated by $\Delta \cong W$, to the generators of a 3 -dimensional subspace of $\left(f_{\text {Klein }}^{\perp}\right)_{3}^{*}$, as shown in the next diagram:

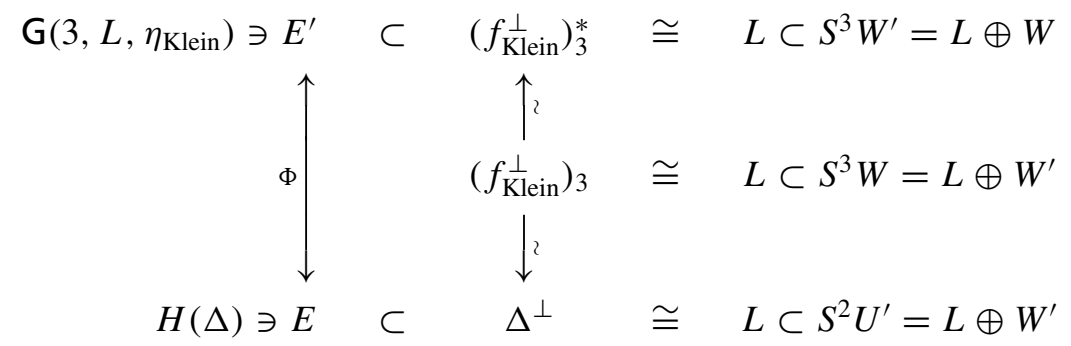

Remark 3.13. The $\mathrm{SL}\left(\mathrm{Z}_{7}\right)$-isomorphism $\Phi$ can now be computed, because by equations (20) (see Appendix A) and (1) we get

$$
\left(v_{1} v_{2} v_{3} \leftrightarrow u_{0}^{2}, v_{2}^{2} v_{3}-v_{1}^{3} \leftrightarrow u_{1} u_{2}, \ldots, v_{3}^{2} v_{2} \leftrightarrow u_{0} u_{2}+u_{3}^{2}\right) .
$$

\section{The six odd 2-torsion points in the $(1,5)$ case}

In this brief section we recall some basic facts on the Horrocks-Mumford bundle $F$ for future comparisons with our results.

The moduli space of Horrocks-Mumford surfaces is given by $\mathrm{P}\left(H^{0}(F)\right)$, where $F$ is the Horrocks-Mumford bundle on $\mathrm{P}^{4}(V)$, cf. [10].

The normaliser of $H_{5}$ is $N=H_{5} \rtimes \mathrm{SL}_{2}\left(Z_{5}\right)$, and $H^{0}(F)$ is irreducible and isomorphic to $\chi_{4}$, the unique 4-dimensional representation of $\mathrm{SL}_{2}\left(Z_{5}\right)$ which factors through $\mathrm{PGL}_{2}\left(Z_{5}\right)$ (for a list of all irreducible $\mathrm{SL}_{2}\left(Z_{5}\right)$-modules see the appendix of [10]). 
Under the involution $\iota\left(x_{i}\right)=x_{-i}$ we get two eigenspaces, and we are interested in $\mathrm{P}_{+}^{1}=\mathrm{P}\left(V_{+}\right)$. Via a suitable action of $\mathrm{SL}_{2}\left(Z_{5}\right)$ on $\mathrm{P}_{+}^{1}$ compatible with the action on $\mathrm{P}^{4}(V)$ one gets that the space $H^{0}\left(\mathcal{O}_{\mathrm{P}_{+}^{1}}(6)\right)$ is a $\mathrm{SL}_{2}\left(\mathrm{Z}_{5}\right)$ module and as such it splits into two irreducible factors of dimension 4 and 3 respectively. More precisely $H^{0}\left(\mathcal{O}_{\mathrm{P}_{+}^{1}}(6)\right) \cong \chi_{4} \oplus \chi_{3}$, and $\chi_{4} \cong H^{0}(F)$. $\mathrm{P}\left(H^{0}\left(\mathscr{O}_{\mathrm{P}_{+}^{1}}(6)\right)\right)$ parametrises sets of six points in $\mathrm{P}_{+}^{1}$, and a surface $X_{s}$ defined by a section $s$ of the Horrocks-Mumford bundle is fully determined by the (unordered) 6-tuple $X_{s} \cap \mathrm{P}_{+}^{1}$. The multiplicities of this 6-tuple also describe the type of surface ([1]): these are listed in Table 2.

TABLE 2. Multiplicities of 2-torsion points in $\mathrm{P}_{+}^{1} \subset \mathrm{P}^{4}(V)$

\begin{tabular}{ll}
\hline multiplicities & type of $X_{s}$ \\
\hline 111111 & abelian surface \\
21111 & translation scroll \\
3111 & tangent scroll \\
222 & double elliptic quintic scroll \\
2211 & union of five quadrics \\
42 & five planes with a double structure \\
\hline
\end{tabular}

We explain these types of surface. If $E$ is a quintic elliptic normal curve $H_{5^{-}}$ equivariantly embedded, then for every point $e \in E$ with $2 e \neq 0$ we define a translation scroll $X$ to be the union of secants

$$
X=\bigcup_{P \in E} \overline{P, P+e} .
$$

The surface $X$ has degree 10 and its singular locus is the curve $E$, where $X$ has transversal $A_{1}$-singularities.

If $e=0$ in the above construction we obtain the tangent scroll of $E$. If $e$ is a non-zero 2 -torsion point then the secants $\overline{P, P+e}$ and $\overline{P, P-e}$ coincide and set-theoretically $X$ is a elliptic quintic scroll. Since $\operatorname{deg} X=10$ the zero locus of the surface supported on $X$ has a double structure.

If finally the elliptic curve $E$ degenerates to a pentagon of lines, the translation scroll degenerates to a union of five quadrics. These can degenerate further to a union of five planes again with double structure.

The multiplicities listed in Table 2 before are just the multiplicities with which the elliptic curve $E$ (as a singular locus in the scrolls) intersects $\mathrm{P}_{+}^{1}$.

REMARK 4.1. It seems reasonable to bear in mind this strategy in our case $p=7$. Indeed we have just seen that $H(\Delta) \cong \operatorname{VSP}(Q, 6)$. In what follows 
we will comment our results with several remarks in order to compare them with the features of this nice case.

\section{Degenerations}

In this section we are going to work out the surface associated to a given boundary point $[\alpha] \in B$ of the moduli space $H(\Delta)$, where $\alpha$ is a matrix in $M$. Furthermore we will relate degenerations of twisted cubic curves (i.e. elements of $H(\Delta)$ ) with degenerations of six general points in the variety of sum of powers of $Q$.

\subsection{Existence of surfaces related to degenerations}

Here we are going to prove that given any element $[\alpha] \in B$ we can find a resolution as in Theorem 2.1.

Proposition 5.1. For any $\alpha$ with $[\alpha] \in B$ we can construct a complex

$$
\begin{aligned}
0 \longleftarrow \mathscr{O}_{A} \longleftarrow \mathcal{O} \longleftarrow & 3 V_{4} \mathcal{O}(-3) \\
\stackrel{\alpha}{\longleftarrow} & 2 S \Omega^{3} \stackrel{\alpha^{\prime}}{\longleftarrow} 3 V_{1} \mathscr{O}(-4) \stackrel{\beta^{\prime}}{\longleftarrow} \mathscr{O}(-7) \longleftarrow 0 .
\end{aligned}
$$

Proof. In the light of Remark 2.3, we get from appendix A that

$$
\operatorname{Hom}_{G_{7}}\left(V_{4} \mathscr{O}(-3), \mathscr{O}\right)=I \oplus U^{\prime} \oplus L,
$$

so the map $\beta$ is naturally given by the three minors $\left(q_{1}(\alpha), q_{2}(\alpha), q_{3}(\alpha)\right)$ of $\alpha$, because by Proposition 2.4 the condition $\Delta$ implies

$$
\left\{q_{1}(\alpha), q_{2}(\alpha), q_{3}(\alpha)\right\} \subset L \subset S^{2} U^{\prime} .
$$

We can then write a sequence exactly like (15), and the $N$-homomorphism in (20) between the copy of the $\mathrm{SL}_{2}\left(\mathrm{Z}_{7}\right)$-module $L \subset S^{2} U^{\prime}$ and $L \otimes V_{4} \subset$ $S^{3} V_{3}=\left(I \oplus U^{\prime} \oplus L\right) \otimes V_{4}$ yields the 21 cubic generators of a variety $A_{\alpha}$ associated to a given $[\alpha] \in H(\Delta)$, when we view $H(\Delta)$ as the space of twisted cubic curves in $\mathrm{P}^{3}(U)$.

The sequence (15) is indeed a complex: $\beta \alpha=0$ can be computed (carefully and patiently) using the exactness of (2), the fact that $\wedge^{3} V \otimes \mathscr{O}(-4) \stackrel{\sigma}{\longrightarrow} \Omega^{3}$ is surjective and so implies

$$
\alpha\left(2 S \Omega^{3}\right)=\alpha\left(2 \sigma\left(\wedge^{3} V \otimes \mathscr{O}(-4)\right)\right),
$$

and the computations of the compositions of these maps, namely $\lambda, \mu$ and $v$ (see Section 2.3). An example: consider the first syzygy $\alpha^{1}$, that is, the first column 
of $\left(a u_{0}^{\#}, b u_{0}^{\#}, c u_{0}^{\#}\right)^{t}=\left(2 a_{0} x_{0}, a_{1} x_{4}, a_{2} x_{1}, a_{3} x_{5}, \ldots\right)^{t}$ from the composition $\alpha \sigma$; for generality assume $a_{0} \neq 0$. The composition $\beta \alpha^{1}$ is a polynomial of degree 4 . Then the coefficient of, e.g., $x_{0} x_{1}^{2} x_{5}$ in $\beta \alpha^{1}$ is given by

$$
\begin{gathered}
2 a_{0}\left(b_{0} e_{2}+b_{2} e_{0}-c_{0} d_{2}-c_{2} d_{0}\right)+a_{2}\left(b_{0} e_{0}-c_{0} d_{0}\right)+a_{3}\left(b_{0} e_{3}+b_{3} e_{0}-c_{0} d_{3}-c_{3} d_{0}\right) \\
-2 b_{0}\left(a_{0} e_{2}+a_{2} e_{0}\right)-b_{2}\left(a_{0} e_{0}\right)-b_{3}\left(a_{0} e_{3}+a_{3} e_{0}\right) \\
+2 c_{0}\left(a_{0} d_{2}+a_{2} d_{0}\right)+c_{2}\left(a_{0} d_{0}\right)+c_{3}\left(a_{0} d_{3}+a_{3} d_{0}\right) .
\end{gathered}
$$

By the condition $\Delta$ the coefficient of $u_{0} u_{2}$ equals that of $u_{3}^{2}$, so this equals

$$
\begin{gathered}
a_{0}\left(b_{0} e_{2}+b_{2} e_{0}-c_{0} d_{2}-c_{2} d_{0}\right)+a_{0}\left(b_{3} e_{3}-c_{3} d_{3}\right) \\
+a_{2}\left(b_{0} e_{0}-c_{0} d_{0}\right)+a_{3}\left(b_{0} e_{3}+b_{3} e_{0}-c_{0} d_{3}-c_{3} d_{0}\right) \\
-b_{0}\left(a_{0} e_{2}+a_{2} e_{0}\right)-b_{0}\left(a_{3} e_{3}\right)-b_{2}\left(a_{0} e_{0}\right)-b_{3}\left(a_{0} e_{3}+a_{3} e_{0}\right) \\
+c_{0}\left(a_{0} d_{2}+a_{2} d_{0}\right)+c_{0}\left(a_{3} d_{3}\right)+c_{2}\left(a_{0} d_{0}\right)+c_{3}\left(a_{0} d_{3}+a_{3} d_{0}\right)
\end{gathered}
$$

which is zero.

Clearly $\beta \alpha=0$ implies $\alpha^{\prime} \beta^{\prime}=0$, and finally the $\Delta$ condition on $\alpha$ guarantees that the composition $\alpha \alpha^{\prime}$ equals zero (on this last fact, see [16], Proposition 3.5).

For the next proofs we are going to use the following criterion for exactness.

Theorem 5.2 ([4] Theorem 20.9). Let $R$ be a ring. A complex

$$
0 \longrightarrow F_{n} \stackrel{\phi_{n}}{\longrightarrow} F_{n-1} \longrightarrow \cdots \longrightarrow F_{1} \stackrel{\phi_{1}}{\longrightarrow} F_{0}
$$

of free $R$-modules is exact if and only if

(1) $\operatorname{rank} F_{k}=\operatorname{rank} \phi_{k}+\operatorname{rank} \phi_{k+1}$, and

(2) depth $I\left(\phi_{k}\right) \geq k$

for $k=1, \ldots, n$.

The notation $I\left(\phi_{k}\right)$ stands for the ideal generated by the minors of $\phi_{k}$, of dimension equal to rank $\phi_{k}$. By [4], Theorem 18.7, we can use the codimension of the variety determined by $I\left(\phi_{k}\right)$ in place of its depth.

In order to prove the exactness of the complex (15) we need to prove first another interesting result. We want to exploit the fact that the matrix (3) determines a sub-morphism

$$
2 V_{4} \mathcal{O}(-3) \stackrel{\left(\begin{array}{l}
d \\
e
\end{array}\right)}{\longleftarrow} S \Omega^{3} .
$$


More precisely this comes from the complex (15) determined by a boundary point $[\alpha] \in H(\Delta)$ :

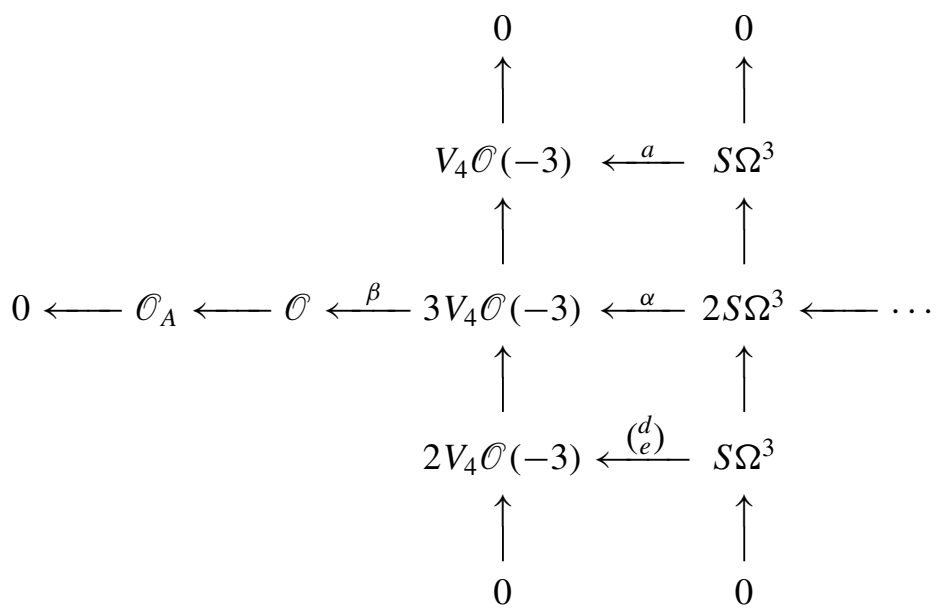

Notice that all the boundary points which are images of orbits with a matrix with a top-left entry like $a$ will admit the sub-morphism as above. More precisely, starting off with the ideal $I_{a}$ generated by the 14 cubics defined by the minors $q_{2}(\alpha)$ and $q_{3}(\alpha)$, we can extract from the complex (15) the following one:

$$
0 \longleftarrow I_{a} \longleftarrow 2 V_{4} \mathcal{O}(-3) \stackrel{\left(\begin{array}{l}
d \\
e
\end{array}\right)}{\longleftarrow} S \Omega^{3} \stackrel{a}{\longleftarrow} V_{1} \mathcal{O}(-4) \longleftarrow 0 .
$$

Proposition 5.3. Let $a \in \Gamma$. Then the complex (16) defined by the ideal $I_{a}$ is exact, and therefore defines a variety $\mathcal{U}_{a}$ of dimension 3 and degree 7.

Proof. Since $I_{a}$ is not contained in any hyperplane we can localise at $x_{i} \neq 0$, where $\Omega^{3}$ is free. To test condition (1) of Theorem 5.2, we first notice that rank $2 V_{4} \mathscr{O}(-3)=14$ and $\operatorname{rank} S \Omega^{3}=20$. Since $\alpha \in \mathrm{P}(M)^{s}$, all the cubics are non-zero, and then the leftmost map has rank 1 , and trivially the variety the 14 cubics determine is of non-zero codimension. Now we need $\operatorname{rank}\left(\begin{array}{l}d \\ e\end{array}\right)=13$ and $\operatorname{rank} a=7$.

There is an injective map $\iota: \Omega^{3} \hookrightarrow \wedge^{3} V^{*} \otimes \mathscr{O}(-3)$ (the quotient is $\Omega^{2}$ ), and hence rank $a\left(V_{1} \mathcal{O}(-4)\right)=\operatorname{rank} \iota\left(a\left(V_{1} \mathscr{O}(-4)\right)\right)$. From this it follows that

$$
\left(\begin{array}{l}
d \\
e
\end{array}\right)\left(\Omega^{3}\right)=\left(\begin{array}{l}
d \\
e
\end{array}\right)\left(\sigma\left(\wedge^{3} V \otimes \mathscr{O}(-4)\right)\right) .
$$

Now let $a \neq d \in \Gamma \subset \mathrm{P}^{3}\left(U^{\prime}\right)$ (see Proposition 3.8), and let $w_{d} \in U^{\prime}$ be such that the composition $d w_{d}=0$. Then we can find a submatrix $\eta$ of 
$\left(\begin{array}{l}d \\ e\end{array}\right)\left(\sigma\left(\wedge^{3} V \otimes \mathscr{O}(-4)\right)\right)$ of the form

$$
\eta:=\left(\begin{array}{cc}
d u_{0}^{\#} & 0 \\
* & e w_{d}
\end{array}\right)
$$

From the computations of $\lambda, \mu$ and $\nu$, we get that $d u_{0}^{\#}=2 a_{0} \operatorname{diag}\left(x_{0}, \ldots, x_{6}\right)+$ $a_{1} v^{*}+a_{2} \lambda^{*}+a_{3} \mu^{*}$ has maximal rank, and since the Pfaffians of $e w_{d}$ are nonzero, we conclude that rank $\left(\begin{array}{l}d \\ e\end{array}\right)=13$.

The variety determined by the $13 \times 13$ minors is contained in the union of the variety cut out by the Pfaffians of $e w_{d}$, which is a 3 -fold, and the one cut out by the determinants of all the top left blocks of rank 7 . The proof that the latter is of dimension at most 3 is precisely as in the following test for the exactness at the map $a$, after replacing $d$ with $a$.

We have

$$
\iota\left(a\left(V_{1} \mathcal{O}(-4)\right)\right)=\left(u_{0}^{\#} a\left|u_{0} a\right| u_{1} a\left|u_{2} a\right| u_{3} a\right)
$$

and the block $u_{0}^{\#} a$ has maximal rank as before. As for the second condition in Theorem 5.2, notice that the Pfaffian varieties $V_{u_{i} a}$ determined by $u_{i} a$, $i=0, \ldots, 3$, are 3-dimensional. Now let $x \in \mathrm{P}^{6}(V)$ be such that $x \notin V_{u_{i} a}$ for some $i$, but $x$ belongs to the variety $Z$ determined by the $7 \times 7$ minors of $\iota\left(a\left(V_{1} \mathcal{O}(-4)\right)\right)$, which is clearly symmetric with respect to $G_{7}$. This means that there is a $j \in\{1, \ldots, 7\}$ such that $\operatorname{Pfaff}_{j}\left(u_{i} a\right)(x) \neq 0$. By $G_{7}$-symmetry we can assume that $j=1$, so there is a linear combination of columns of $u_{i} a(x)$ such that it is equivalent to

$$
g(x):=\left(\begin{array}{ll}
0 & * \\
0 & T
\end{array}\right)
$$

where $T$ is an invertible matrix. If $a$ is one of the three elements with 0 -entries, we can compute by hand that after rearranging the columns of $\iota\left(a\left(V_{1} \mathcal{O}(-4)\right)\right)$ we get upper triangular matrices with the seven $x_{i}$ 's as entries. Taking suitable columns we see that the intersection of these determinants is contained in projective subspaces of codimension 3 .

So let $a$ have all the entries non-zero, and take a column $k$ of $u_{0}^{\#} a$ such that the top entry $k_{1}(x) \neq 0$. Substituting $k(x)$ in place of the first column of $g(x)$ we get that the rank is maximal, a contradiction. Therefore $Z \subset$ $V_{u_{0} a} \cup V_{u_{1} a} \cup V_{u_{0} a} \cup V_{u_{1} a}$, so $\operatorname{dim}(Z) \leq 3$ and the complex is exact.

Finally, the Hilbert polynomial of $I_{a}$ : let $K$ be the kernel of $I_{a} \leftarrow 2 V_{4} \mathscr{O}(-3)$ as a map of direct sums of line bundles. Then, exactly as in [16], Theorem 2.5, by "kind of adding" a piece of the Koszul complex to the resolution (16), we 
obtain the following commutative exact diagram.

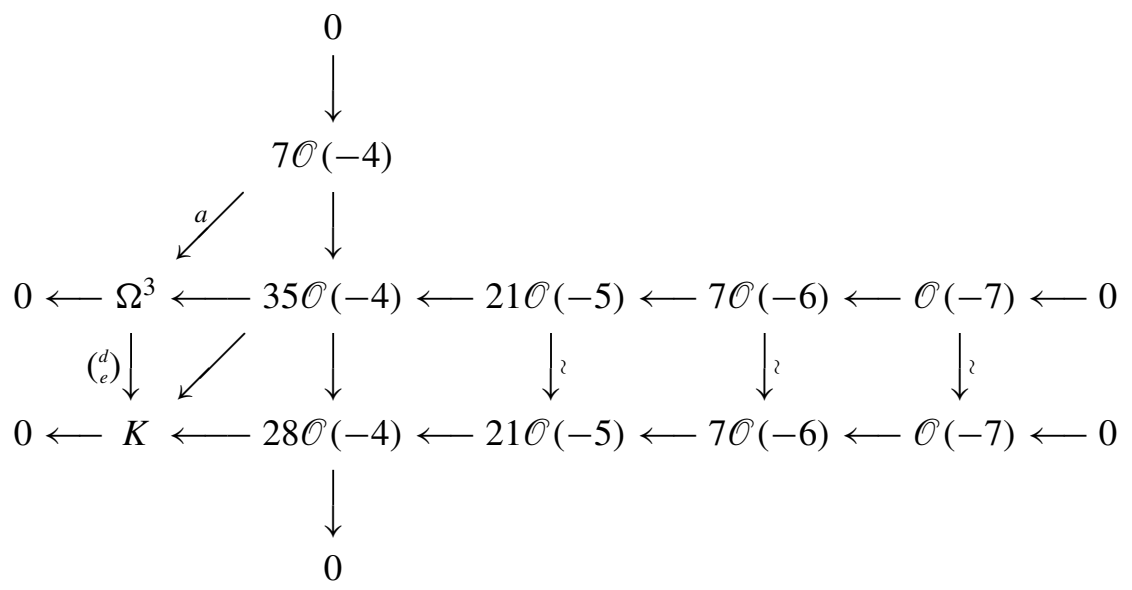

The bottom row leads to a resolution of $\mathscr{U}_{a}$ involving only direct sums of line bundles, namely

$$
\begin{aligned}
0 \longleftarrow I_{\mathscr{U}_{a}} \longleftarrow 14 \mathscr{O}(-3) & \longleftarrow 28 \mathscr{O}(-4) \\
& \longleftarrow 21 \mathscr{O}(-5) \longleftarrow 7 \mathscr{O}(-6) \longleftarrow \mathscr{O}(-7) \longleftarrow 0
\end{aligned}
$$

Now we can compute the Hilbert polynomial of $\mathscr{U}_{a}$, from which the result follows: $P_{\mathscr{U}_{a}}=\frac{7}{6} n^{3}+\frac{7}{2} n^{2}+\frac{7}{3} n$.

Thanks to this intermediate result we can prove now the main proposition of this subsection:

Proposition 5.4. The complex (15) is exact. Therefore any $\alpha$ with $[\alpha] \in B$ defines a variety $A_{\alpha}$ of dimension 2 and degree 14.

Remark 5.5. We are not claiming that the statement of Proposition 5.4 holds for every $[\alpha] \in H(\Delta)$, although we know by [16] that for a general point of $H(\Delta)$ the complex is exact.

Proof. We have restricted our attention to the case when $\alpha$ represents an element of $B \subset H(\Delta)$, which we write as in (3). As before, $A_{\alpha}$ is not contained in any hyperplane, so we can localise at $x_{i} \neq 0$, where $\Omega^{3}$ is free.

To test the first condition from Theorem 5.2, notice that rank $3 V_{4} \mathcal{O}(-3)=$ 21 and rank $2 S \Omega^{3}=40$. Since $\alpha \in \mathrm{P}(M)^{s}, \beta \neq 0$, so rank $\beta=1$, and thus the only thing we need is rank $\alpha=20$. Observe that rank $\alpha=\operatorname{rank} \alpha^{\prime}$, in fact from the injective map $\Omega^{3} \stackrel{\iota}{\longleftrightarrow} \wedge^{3} V^{*} \otimes \mathscr{O}(-3)$, using the definition of $\alpha^{\prime}$ and 
the fact that the entries of $\alpha^{\prime}$ are just transposes of the entries of $\alpha$, we get

$$
\begin{aligned}
\operatorname{rank} \alpha^{\prime}\left(3 V_{1} \mathscr{O}(-4)\right) & =\operatorname{rank} 2 \iota\left(\alpha^{\prime}\left(3 V_{1} \mathscr{O}(-4)\right)\right) \\
& =\operatorname{rank}\left(\alpha\left(2 \sigma\left(\wedge^{3} V \otimes \mathscr{O}(-4)\right)\right)\right)^{t} .
\end{aligned}
$$

So once we show rank $\alpha=20$ we shall have proved the first condition for the rest of the sequence as well.

Let $\alpha_{22}=d \in \Gamma \subset \mathrm{P}^{3}\left(U^{\prime}\right)$ (see Proposition 3.8), and let $a \neq w_{d} \in U^{\prime}$ be such that the composition $d w_{d}=0$. Then we can find a submatrix $\tilde{\alpha}$ of $\alpha\left(2 \sigma\left(\wedge^{3} V \otimes \mathcal{O}(-4)\right)\right)$ of the form

$$
\tilde{\alpha}:=\left(\begin{array}{ccc}
a u_{0}^{\#} & 0 & 0 \\
* & d u_{0}^{\#} & 0 \\
* & * & e w_{d}
\end{array}\right) .
$$

From the computations of $\lambda, \mu$ and $\nu$, we get that $a\left(u_{0}^{\#}\right)$ and $d\left(u_{0}^{\#}\right)$ have maximal rank, and since the Pfaffians of $e w_{d}$ are non-zero, we conclude that $\operatorname{rank} \alpha=20$.

To test the second condition, first notice that any product of two Pfaffians of an antisymmetric matrix like $e w_{d}$ can be computed as the determinant of a suitable $6 \times 6$ minor. We need to prove two things: that depth $I_{\beta^{\prime}}\left(=\operatorname{depth} I_{\beta}\right) \geq$ 4 and depth $I_{\alpha^{\prime}}\left(=\right.$ depth $\left.I_{\alpha}\right) \geq 3$. For the latter part, observe that we can extract matrices like $\tilde{\alpha}$ from $\alpha$, but with the top and middle diagonal blocks being given by columns as in the proof of Proposition 5.3 about the codimension of $Z$ determined by $a$. Therefore the variety $Z^{\prime}$ determined by the maximal rank minors of $\alpha$ is contained in the union of the varieties determined by the three blocks (which we can vary), whose codimension is at least 3 .

For $\beta^{\prime}$, with the usual matrix, notice that by Proposition 5.3 the middle and bottom $V_{4}$ 's determine a threefold of degree 7. Because $\alpha$ is stable, the 21 cubics are independent, and symmetric with respect to $G_{7}$. Therefore all the syzygies of a resolution of $A_{\alpha}$ are $G_{7}$-modules, namely 7-dimensional vector spaces in general, except when the syzygies have degree a multiple of 7. But in that case $\operatorname{dim} S^{7 n} V^{*} \equiv 1$ modulo 7, and because the sum (with suitable sign) of those syzygies has to be precisely -1 , the Hilbert polynomial of $A_{\alpha}$ is divisible by 7 . Now consider the variety determined by all the 21 cubics. Because the resolution of $\mathscr{U}_{a}$ has length precisely three, it does not contain a lower dimensional (possibly embedded) component. In fact, if there was one, call it $Z_{0}$ and let $I_{Z_{0}}$ and $I_{\hat{Z}_{0}}$ be the ideals of $Z_{0}$ and of the union of the rest of the components. Then $I_{a}=I_{Z_{0}} \cdot I_{\hat{Z}_{0}}$, and therefore a resolution of $I_{a}$ would be at least as long as the resolution of $Z_{0}$, namely at least 4 . This leads to the fact that when we add the top 7 cubics to the 14 generating $\mathscr{U}_{a}$, the new variety we get, that is $A_{\alpha}$, has to drop dimension, otherwise the degree 
would drop to some number not a multiple of 7 . Hence we have proved that depth $I_{\beta^{\prime}}=$ depth $I_{\beta} \geq 4$.

The part on the degree and the dimension is straightforward once we notice that it holds for general $(1,7)$-polarised abelian surfaces, and that the shape of the resolution determines the Hilbert polynomial of the variety, which is a constant feature on the varieties parametrised by $H(\Delta)$.

Remark 5.6. By equation (10) we know that for a general $(1,7)$-polarised abelian variety the six points in $W^{\prime}$ in Proposition 2.9 must be the preimages of

$$
\left.\left\langle q_{1}(\alpha), q_{2}(\alpha), q_{3}(\alpha)\right\rangle \cap\left(W^{\prime}\right)^{3}\right|_{L} \subset L .
$$

But $\left\langle q_{1}(\alpha), q_{2}(\alpha), q_{3}(\alpha)\right\rangle$ determines the 4-dimensional subspace of $L \subset S^{3} W$ given by the minors of $\psi$ (see equation (11)), and clearly we get that the space spanned by the third powers of the (possibly degenerate) six points restricted to $L$ must be contained in, and generally equal to, $\left\langle q_{1}(\alpha), q_{2}(\alpha), q_{3}(\alpha)\right\rangle$.

\subsection{General degenerations}

So far we have proved that for all the varieties $A_{\alpha}$ parameterised over the same $a \in \Gamma \subset \mathrm{P}^{3}(U)$ (see Proposition 3.8) we get $A_{\alpha} \subset \mathscr{U}_{a}$. Again by Proposition 3.8 we also know that this occurs when the twisted cubic curve in $\mathrm{P}^{3}(U)$ defined by $\alpha$ is degenerate. Now we want to find out what this result means in terms of the other descriptions of $H(\Delta)$, and specifically $\operatorname{VSP}(Q, 6)$. As usual we work with (3).

Proposition 5.7. Let $A_{\alpha}$ be the variety associated to an element $[\alpha] \in B \subset$ $H(\Delta)$, and let $A_{\alpha} \cap \mathrm{P}^{2}\left(W^{\prime}\right)=\left\{p_{1}, \ldots, p_{6}\right\}$. Then three of the six $\left.p_{i}^{3}\right|_{L} \in L$, say $p_{1}, p_{2}, p_{3}$, lie on a line and therefore, in contrast to Proposition 2.9, the ideal of $A_{\alpha}$ is not generated by the Pfaffians defining $V_{7, p_{1}}, V_{7, p_{2}}$ and $V_{7, p_{3}}$.

Proof. Let $w \notin\langle a\rangle$ be an element of $\operatorname{Hom}_{G_{7}}\left(V_{1} \mathscr{O}(-4), S \Omega^{3}\right)=U^{\prime}$, that is, a linear combination of $\left\langle u_{0}, u_{1}, u_{2}, u_{3}\right\rangle$, such that $e^{*} w=0$ (see Remark 3.6). This requirement is non-trivial, because in general $\operatorname{rank}\left(e^{*}\right)=3$, therefore such a $w$ does not exist. Nevertheless in the proof of Proposition 3.8 we saw that for a given $a$ we can find in general three $e$ 's such that $a^{*} e=0$ and $\operatorname{rank}\left(e^{*}\right)=2$ (precisely when $e \in \Gamma$ ).

From the Koszul complex of $\Omega^{3}$ we get that $35 \mathcal{O}(-4)$ maps surjectively to $S \Omega^{3}$, and we can consider the syzygies generated by the composition of $w$ and $\left(\begin{array}{l}d \\ e\end{array}\right)$. In other words we get that $\left(\begin{array}{l}d \\ e\end{array}\right)\left(S \Omega^{3}\right)$ contains a column like

$$
\left(\begin{array}{l}
d \\
e
\end{array}\right)(w)=\left(\begin{array}{c}
d^{*} w \\
e^{*} w
\end{array}\right)=\left(\begin{array}{c}
d^{*} w \\
0
\end{array}\right)
$$


If $A_{\alpha}$ is the degenerate $(1,7)$-polarised abelian surface associated to $\alpha$, then the above column is nothing but 7 of the first 49 linear syzygies of the 21 cubics that define it. Bearing in mind Proposition 2.8 and the fact that $\alpha_{12}=0$, we get that the middle $V_{4}$ that generates the ideal of $A_{\alpha}$ must be given by the 7 principal Pfaffians of

$$
\begin{aligned}
M_{7}^{\prime}\left(x, d^{*} w\right)= & \left(w_{0} d_{3}+w_{3} d_{0}-d_{1} w_{1}\right) \mu \\
& +\left(w_{0} d_{1}+w_{1} d_{0}-d_{2} w_{2}\right) v+\left(w_{0} d_{2}+w_{2} d_{0}-d_{3} w_{3}\right) \lambda .
\end{aligned}
$$

Notice that this does not depend on $w$; in fact $\left\{x \in \mathrm{P}^{3}\left(U^{\prime}\right) \mid e^{*} x=0\right\}=$ $\langle a, w\rangle$, thus $d^{*}(\xi a+\psi w)=\xi d^{*} a+\psi d^{*} w=0+\psi d^{*} w$. Furthermore $d^{*} w \neq(0: 0: 0)$, because even if $\operatorname{rank}\left(d^{*}\right)=2,\left\{x \in \mathrm{P}^{3}\left(U^{\prime}\right) \mid d^{*} x=0\right\}=$ $\left\langle a, w^{\prime}\right\rangle \not \supset w$, otherwise $e=d$. Observe that, by Proposition 5.4, $\left.\left(d^{*} w\right)^{3}\right|_{L}=$ ae.

The crucial observation is that because in general there are three such points $e \in \Gamma$, call them $e, e^{\prime}, e^{\prime \prime}$, it is possible to find three corresponding sets of seven cubic (Pfaffian) generators of $A_{\alpha}$ as above. Therefore, for a map like

$$
\mathcal{O} \longleftarrow 2 V_{4} \mathscr{O}(-3) \stackrel{\left(\begin{array}{l}
d \\
e
\end{array}\right)}{\longleftarrow} S \Omega^{3}
$$

to exist, one $d^{*} w_{i}, i \in\left\{e, e^{\prime}, e^{\prime \prime}\right\}$ has to be a linear combination of the others. But this is obvious by Remark 5.6, because

$$
\operatorname{dim}\left\langle\left.\left(d^{*} w_{e}\right)^{3}\right|_{L},\left.\quad\left(d^{*} w_{e^{\prime}}\right)^{3}\right|_{L},\left.\quad\left(d^{*} w_{e^{\prime \prime}}\right)^{3}\right|_{L}\right\rangle=\operatorname{dim}\left\langle a e, a e^{\prime}, a e^{\prime \prime}\right\rangle=2 .
$$

REMARK 5.8. After tensoring with $V_{4}$, the three collinear elements $\left.\left(d^{*} w_{e}\right)^{3}\right|_{L},\left.\left(d^{*} w_{e^{\prime}}\right)^{3}\right|_{L}$ and $\left.\left(d^{*} w_{e^{\prime \prime}}\right)^{3}\right|_{L}$ of $L$ clearly generate the ideal of the variety $\mathscr{U}_{a}$ of Proposition 5.3.

Remark 5.9. By Proposition 5.3 and the previous Remark 5.8, we see that $\mathcal{U}_{a}$ must be contained in $V_{7, d^{*} w_{e}} \cap V_{7, d^{*} w_{e^{\prime}}} \cap V_{7, d^{*} w_{e^{\prime \prime}}} \subset \mathrm{P}^{6}(V)$. Because $\mathscr{U}_{a}$ and the $V_{7, d^{*} w_{i}}$ are all 3-dimensional, we conclude that the $V_{7, d^{*} w_{i}}$ are either nonreduced, or reducible. In the former case, by Proposition 2.8(ii) the preimages of the $d^{*} w_{i}$ are not general points of $\mathrm{P}^{2}\left(W^{\prime}\right)$.

Clearly at this stage information about the nature of the three points $d^{*} w_{e}$, $d^{*} w_{e^{\prime}}, d^{*} w_{e^{\prime \prime}} \in W^{\prime}$ must be supplied. Therefore in the next proposition we are going to give a (slightly) computational analysis of the general picture we are dealing with.

Proposition 5.10. Let $\mathscr{U}_{a}$ be as in Proposition 5.3. Then the three points $d^{*} w_{e}, d^{*} w_{e^{\prime}}$ and $d^{*} w_{e^{\prime \prime}} \in W^{\prime}$ lie on the curve $y_{1}^{5} y_{3}+y_{2}^{5} y_{1}+y_{3}^{5} y_{2}-5 y_{1}^{2} y_{2}^{2} y_{3}^{2}=$ $\operatorname{Hess}\left(y_{1}^{3} y_{2}+y_{2}^{3} y_{3}+y_{3}^{3} y_{1}\right)=0$, the Hessian of the Klein quartic $f_{\mathrm{Klein}}^{\prime}$ in $\mathrm{P}^{2}\left(W^{\prime}\right)$. 
Proof. What follows is restricted to the open part $\mathrm{P}^{2}(W)_{0}:=\left\{\left(v_{1}: v_{2}\right.\right.$ : $\left.\left.v_{3}\right) \in \mathrm{P}^{2}(W) \mid v_{1} v_{2} v_{3} \neq 0\right\}$. In this way we lose three points of $Q$ which, however, will be completely treated in the next subsection.

$Q$ and $\Gamma$ are isomorphic, so let $\left(v_{1}: v_{2}: v_{3}\right) \in Q \subset \mathrm{P}^{2}(W)_{0}$; then we get

$$
a=\left(1: \frac{v_{1}}{v_{3}}: \frac{v_{2}}{v_{1}}: \frac{v_{3}}{v_{2}}\right)=\left(v_{1} v_{2} v_{3}: v_{1}^{2} v_{2}: v_{2}^{2} v_{3}: v_{3}^{2} v_{1}\right) \in \Gamma,
$$

namely a top-left entry of a matrix $\alpha \in \phi^{-1}(B)$.

Let $\left\{x \in \mathrm{P}^{3}\left(U^{\prime}\right) \mid a^{*} x=0\right\}=\langle e, d\rangle$. To simplify the computations we assume $d_{0}=0$; then we get

$$
d=\left(0: \frac{1}{v_{1}}: \frac{1}{v_{2}}: \frac{1}{v_{3}}\right)=\left(0: v_{2} v_{3}: v_{1} v_{3}: v_{2} v_{1}\right)
$$

and we may put $e=\left(1: e_{1}: e_{2}: e_{3}\right)$. As in the proof of Proposition 3.8, assume $e \in \Gamma$. We saw in Lemma 3.10 that in general there are three such $e$ s. Notice that this means that $\langle e, d\rangle$ is a trisecant of $\Gamma$ in $\mathrm{P}^{3}\left(U^{\prime}\right)$. Let $\{x \in$ $\left.\mathrm{P}^{3}\left(U^{\prime}\right) \mid e^{*} x=0\right\}=\langle a, w\rangle$ with $w_{0}=0$. Then the point of $\mathrm{P}^{2}\left(W^{\prime}\right)_{0}$ which yields $e a \otimes V_{4}$ up to scalar, as in Proposition 2.8 , is given by $d^{*} w=\left(d_{3} w_{3}\right.$ : $\left.d_{1} w_{1}: d_{2} w_{2}\right):=\left(y_{1}: y_{2}: y_{3}\right)$. We are abusing the notation, because actually we should be working with elements of $W^{\prime}$, not its projectivisation. In other words

$$
e a \otimes V_{4}=I_{3}\left(d_{3} w_{3}: d_{1} w_{1}: d_{2} w_{2}\right) \text {. }
$$

Hence $e=\left(1: \frac{y_{1} v_{3}}{y_{2} v_{1}}: \frac{y_{2} v_{1}}{y_{3} v_{2}}: \frac{y_{3} v_{2}}{y_{1} v_{3}}\right)$.

If $e$ is a solution of $a^{*}$, then

$$
0=a^{*} e=\left(\begin{array}{c}
y_{2} y_{3} v_{1}^{2}+y_{3} y_{1} v_{3}^{2}-y_{2}^{2} v_{3} v_{1} \\
y_{3} y_{1} v_{2}^{2}+y_{1} y_{2} v_{1}^{2}-y_{3}^{2} v_{1} v_{2} \\
y_{1} y_{2} v_{3}^{2}+y_{2} y_{3} v_{2}^{2}-y_{1}^{2} v_{2} v_{3} .
\end{array}\right)=\left(\begin{array}{c}
\sigma_{1} \\
\sigma_{2} \\
\sigma_{3}
\end{array}\right) .
$$

Let $S$ be the variety in $\mathbf{P}^{2}(W) \times \mathbf{P}^{2}\left(W^{\prime}\right)$ defined by $\sigma_{1}, \sigma_{2}$ and $\sigma_{3}$, and consider the projections

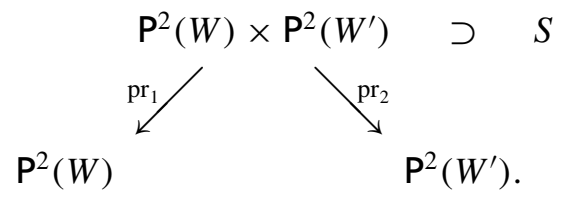

It is easy to see that $v_{1} v_{3} y_{3} \sigma_{1}+v_{1} v_{2} y_{1} \sigma_{2}+v_{2} v_{3} y_{2} \sigma_{3}=y_{1} y_{2} y_{3}\left(v_{1}^{3} v_{2}+v_{2}^{3} v_{3}+\right.$ $\left.v_{3}^{3} v_{1}\right)$, and therefore $\operatorname{pr}_{1}\left(S \cap \mathrm{P}^{2}(W)_{0} \times \mathrm{P}^{2}\left(W^{\prime}\right)_{0}\right)=Q \cap \mathrm{P}^{2}(W)_{0}$. Furthermore $\mathrm{pr}_{1}$ is generically $3: 1$. 
In the same way, but through a computation with Maple, we see that

$$
v_{1}^{2} v_{2}^{2} v_{3}^{2} y_{3}^{3} y_{2}\left(y_{1}^{5} y_{3}+y_{2}^{5} y_{1}+y_{3}^{5} y_{2}-5 y_{1}^{2} y_{2}^{2} y_{3}^{2}\right)^{2} \in I\left(\sigma_{1}, \sigma_{2}, \sigma_{3}\right):
$$

thus $\operatorname{pr}_{2}\left(S \cap \mathrm{P}^{2}(W)_{0} \times \mathrm{P}^{2}\left(W^{\prime}\right)_{0}\right)=\left\{y_{1}^{5} y_{3}+y_{2}^{5} y_{1}+y_{3}^{5} y_{2}-5 y_{1}^{2} y_{2}^{2} y_{3}^{2}=0\right\} \cap$ $\mathrm{P}^{2}\left(W^{\prime}\right)_{0}$.

Observe that the initial restriction to $\mathrm{P}^{2}(W)_{0}$ does not change the result: in fact starting with $(1: 0: 0) \in W$ we get the elements $(0: 1: 0)$, $(0: 0: 1) \in \operatorname{Hess}\left(Q^{\prime}\right)$, and by the usual action of $\mathrm{PGL}_{2}\left(\mathrm{Z}_{7}\right)$ on $Q$ this is enough.

Remark 5.11. The construction of the last proof associates to every point of $Q \subset \mathrm{P}^{2}(W)$ three points of Hess $\left(Q^{\prime}\right)$ which we know are determined by $a$ and the trisecant of $\Gamma \subset \mathrm{P}^{3}\left(U^{\prime}\right)$ via the product $*$. Then, by Proposition 5.7, the projective classes of their third power restricted to $L$ lie on a trisecant of $\left.\mathrm{P}^{2}\left(W^{\prime}\right)^{3}\right|_{L} \subset \mathrm{P}^{6}(L)$.

Moreover observe that $\operatorname{pr}_{2}(S)$ is, by construction, a $\mathrm{PGL}_{2}\left(\mathrm{Z}_{7}\right)$-invariant curve.

The Hessian of $Q^{\prime}$ seemingly carries special information about $V_{7, y} \subset$ $\mathrm{P}^{6}(V)$, for $y \in \mathrm{P}^{2}\left(W^{\prime}\right)$. And in fact for every $y \in \operatorname{Hess}\left(Q^{\prime}\right)$ there is some $v=\operatorname{pr}_{1}\left(\operatorname{pr}_{2}^{-1}(y)\right) \in Q$ that, by the results of this subsection and the previous construction, determines a variety $V_{7, y} \subset \mathrm{P}^{6}(V)$ which is either non-reduced, or reducible.

All this can be summarised in the next proposition.

Proposition 5.12. Let $[\alpha] \in B$ be a general boundary point of $H(\Delta)$, i.e. $[\alpha] \in B \backslash B^{\prime} \subset H(\Delta)$ and a is not a cusp. Then the degenerate $(1,7)$-polarised abelian variety $A_{\alpha}$ associated to $\alpha$ is the intersection of a 3-fold $U_{a}$ of degree 7 and a Calabi-Yau 3-fold. Moreover $\mathcal{U}_{a}$ is generated by three distinct collinear points of $\mathrm{P}^{6}(L)$ tensored by $V_{4}$, images of three points of $\operatorname{Hess}\left(Q^{\prime}\right) \subset \mathrm{P}^{2}\left(W^{\prime}\right)$ uniquely determined by the top-left entry $a \in \Gamma$ of $\alpha$.

Proof. By Remark 5.6 we know that the ideal generated by the minors of $\alpha$ is also generated by the image of six points $\left\{p_{1}, \ldots, p_{6}\right\} \in W^{\prime}$ as in (2.9). By Proposition 5.7 we know that the images in $\mathrm{P}^{6}(L)$ of three of these six points, say $\left\{p_{1}, p_{2}, p_{3}\right\}$ are collinear, and by Remark 5.8 we know that (once tensored by $V_{4}$ ) they generate the ideal of a 3 -fold $\mathscr{U}_{a}$ of degree 7 and are determined by $a$. Clearly we have that $A_{\alpha}=\mathscr{U}_{a} \cap V_{7, p_{i}}$ for any $i \in\{4,5,6\}$. Finally, by Proposition 5.10 we know that $\left\{p_{1}, p_{2}, p_{3}\right\} \subset \operatorname{Hess}\left(Q^{\prime}\right) \subset \mathrm{P}^{2}\left(W^{\prime}\right)$.

Remark 5.13. We contrast Proposition 5.12 with the projective degenerations found in the $(1,5)$ case in $[1]$ and in the $(1,7)$ case in [18]. There the 
general degeneration is a translation scroll over an elliptic curve embedded in a certain $\mathrm{P}^{4}$ (respectively $\mathrm{P}^{6}$ ).

Because our boundary $B$ is birational to the universal Kummer surface that parametrises translation scrolls it is tempting to expect a similar picture here. But an elliptic curve in $\mathrm{P}^{6}(V)$ cannot be determined by $a \in \Gamma \cong Q \subset \mathrm{P}^{2}(W)$, as $\Gamma$ is not isomorphic to the Klein curve in $\mathrm{P}^{2}\left(W^{\prime}\right) \subset \mathrm{P}^{6}(V)$ as a curve with $\mathrm{PGL}_{2}\left(\mathrm{Z}_{7}\right)$-action. Therefore if the degeneration $A_{\alpha}$ were a translation scroll it would be contained in some $V_{7, a^{\prime}}$, where $a^{\prime} \in W^{\prime}$ defines a secant variety over the above elliptic curve, see Proposition 2.8 part (1).

Certainly this does not occur for the general degeneration of our case because, as we saw, the points $\left\{p_{1}, p_{2}, p_{3}\right\}$ as before do not lie in general on the Klein quartic. The only possibility is that such an $a^{\prime}$ can be found among $\left\{p_{4}, p_{5}, p_{6}\right\}$, but not defined by $a$, otherwise the degeneration would be uniquely defined by $a$ and we would end up with the same degeneration for every point of the fibre in $B$ over $a$, which is certainly not the case, because the minors of matrices of distinct orbits in $\mathrm{P}(M)^{s}$ span different 3-spaces of $L$, and therefore once tensored with $V_{4}$ they determine different surfaces in $\mathrm{P}^{6}(V)$. But this would give a second rational map $B \rightarrow Q$ given by $\alpha \mapsto a^{\prime}$, nonconstant on the fibres of $\alpha \mapsto a$. But this is impossible, as the general fibre is rational and $Q$ has genus 3 .

From our point of view, the first degeneration of six points in $\mathrm{P}^{2}\left(W^{\prime}\right)$ is not given by the multiplicity, but by the failure of Proposition 2.9 part (2), namely by the fact that the images of three of the six points in $\mathrm{P}^{6}(L)$ do not span a plane, but a line only.

\subsection{Degenerations arising from $B^{\prime} \subset B$}

At this stage degenerations of this sort are relatively simple to describe. First of all we assume that we are working with an element $[\alpha] \in B^{\prime}$, therefore we can assume that

$$
\alpha=\left(\begin{array}{ll}
a & 0 \\
b & d \\
0 & e
\end{array}\right) .
$$

Furthermore we assume that $a$ is not the image of a cusp of $Q$; that case will be treated in the next subsection.

Proposition 5.14. Let $\alpha$ be as above. Then the degenerate (1, 7)-polarised abelian variety $A_{\alpha}$ it determines is the intersection of two 3-folds $\mathscr{U}_{a}$ and $\mathcal{U}_{e}$ of degree 7. Moreover $\mathcal{U}_{a}$ (resp. $\mathcal{U}_{e}$ ) is generated by three distinct collinear points of $\mathrm{P}^{6}(L)$ tensored by $V_{4}$. These are images of three points $\left\{p_{1}, p_{2}, p_{3}\right\}$ (resp. $\left.\left\{p_{1}, p_{4}, p_{5}\right\}\right)$ of $\operatorname{Hess}\left(Q^{\prime}\right) \subset \mathrm{P}^{2}\left(W^{\prime}\right)$ uniquely determined by the top-left entry $a \in \Gamma$ (resp. bottom-right entry $e \in \Gamma)$ of $\alpha$. 
PROOF. Everything works as in the proof of Proposition 5.12, moreover the same argument holds for the sub-matrix $\left(\begin{array}{l}a \\ b\end{array}\right)$ of $\alpha$ and for the 3 -fold $\mathscr{U}_{e}$ it determines (see Proposition 5.3).

Notice that $U_{e}$ is generated by $e a \otimes V_{4}$ and $e b \otimes V_{4}$, whereas $e a \otimes V_{4}$ and $a d \otimes V_{4}$ generate $\mathscr{U}_{a}$. Therefore $p_{1}$ is the preimage in $W^{\prime}$ of $a e \in L$. Or, if we set $b$ and $d$ to be in $\Gamma$, exactly as in equation (17) we get $p_{1}$ given by $d^{*} b=b^{*} d \in W^{\prime}$.

Finally notice that in place of $V_{7, p_{4}}$ and $V_{7, p_{5}}$ of Proposition 5.12 we have used $\mathscr{U}_{e}$, which is clearly contained in both.

REMARK 5.15. In the notation of this subsection we see that if $a$ and $e$ are general, namely are not cusps of $\Gamma$, then by Lemma 3.10 the five points of Proposition 5.14 are distinct, and so we are left again with the interpretation of the varieties parametrised by $B^{\prime}$. If those were elliptic or tangent scrolls over an elliptic curve $E$, then by Table 2 any of them would intersect $\mathrm{P}^{2}\left(W^{\prime}\right)$ with multiplicity respectively $(2,2,2)$ and $(3,1,1,1)$, which is not the case here.

Moreover, by Proposition 1.1 in [2], we see that an elliptic scroll in $\mathrm{P}^{6}(V)$ contains three elliptic curves, and so if $B^{\prime}$ was the space parameterising elliptic scrolls, a fibre over an element like $a \in \Gamma$ should intersect three suitable distinct fibres at each of the three points of intersection with $B^{\prime}$. This means that the degenerate twisted cubic curve defined by $[\alpha] \in B^{\prime}$ should be the union of three straight lines all meeting at a point. But with a simple argument about a $\mathrm{P}^{1}$ of conics (like those in $\{a=0\} \subset \mathrm{P}^{3}(U)$ defined by the fibre over $a$ ), we get that a case like that cannot occur.

\subsection{Degenerations over cusps}

In this subsection we study the boundary points of $H(\Delta)$ over cusps. As we will see, this can be done by hand, and it will be a good example of how we recover the 21 cubic generators, or more precisely the $3 G_{7}$-modules $V_{4}$, from the syzygies that define a degeneration.

Here we are going to study the fibre of $H(\Delta)$ over (the image in $\Gamma$ of) a specific cusp of $Q$. This is enough because the action of $\mathrm{PGL}_{2}\left(\mathrm{Z}_{7}\right)$ permutes the cusps of $Q$. Notice that the action of $\mathrm{PGL}_{2}\left(\mathrm{Z}_{7}\right)$ on $L$ induces an action on $H(\Delta)$ as well, if we view an element of $H(\Delta)$ as a 3-dimensional subspace of $L$. Alternatively we can take the action of $\mathrm{PGL}_{2}\left(\mathrm{Z}_{7}\right)$ on the entries (in $U^{\prime}$ ) of a matrix $\alpha$ representing a point in $H(\Delta)$.

As usual let $[\alpha] \in B \subset H(\Delta)$, and $\alpha$ be as in (3). We assume that $\alpha_{11}=u_{1}$. Then as before $\langle d, e\rangle=\left\langle u_{2}, u_{3}\right\rangle$, and after setting $d=u_{2}$ and $e=u_{3}$, we get

$$
\alpha=\left(\begin{array}{cc}
u_{1} & 0 \\
\xi u_{3} & u_{2} \\
-\xi u_{0}+\tau u_{3} & u_{3}
\end{array}\right) .
$$


Notice that if $(\xi: \tau)=(0: 1)$, the corresponding matrix, and therefore element of $B$, lies on the intersection of the three $\mathrm{P}^{1}$ 's over the cusps $u_{1}, u_{2}$ and $u_{3}$.

Proposition 5.16. Let $\alpha$ be as in (18). Then the 21 cubics it determines define a variety $A_{\alpha}$ as follows.

(1) If $\xi \neq 0 \neq \tau$ then $A_{\alpha}$ is 7 quadric surfaces, each contained in some $\mathrm{P}^{3} \subset \mathrm{P}^{6}(V)$. As a configuration in $\operatorname{VSP}(Q, 6)$ this corresponds to a double point and two single points on a line in $\mathrm{P}^{2}\left(W^{\prime}\right)$, plus a second double point.

(2)(a) if $\tau=0$ then $A_{\alpha}$ is 7 double planes in $\mathrm{P}^{6}(V)$. Then we get that the related configuration in $\operatorname{VSP}(Q, 6)$ is a quadruple point plus a double point in $\mathrm{P}^{2}\left(W^{\prime}\right)$.

(b) if $\xi=0$ then $A_{\alpha}$ is 14 planes in $\mathrm{P}^{6}(V)$. The related configuration in $\operatorname{VSP}(Q, 6)$ is given by three double points in $\mathrm{P}^{2}\left(W^{\prime}\right)$.

These degenerations were also found by Melliez and Ranestad by a different method: see [18].

Proof. We first compute the 3-dimensional linear subspace $E$ of $L$ spanned by the minors of $\alpha$

$$
E=\left\langle\xi\left(u_{0} u_{2}+u_{3}^{2}\right)-\tau\left(u_{2} u_{3}\right), u_{1} u_{3}, u_{1} u_{2}\right\rangle
$$

or via the $\mathrm{SL}_{2}\left(\mathrm{Z}_{7}\right)$-isomorphism as in Remark 3.13

$$
E=\left\langle\xi\left(v_{2} v_{3}^{2}\right)-\tau\left(v_{3}^{2} v_{1}-v_{2}^{3}\right), v_{1}^{2} v_{2}-v_{3}^{3}, v_{2}^{2} v_{3}-v_{1}^{3}\right\rangle
$$

This amounts to saying that, as shown in the proof of Proposition 5.4, the 21 generators of $A_{\alpha}$, and more precisely the top, middle and bottom $V_{4}$ 's are

$$
\begin{aligned}
V_{4}^{t} & =-\tau\left\{x_{i} x_{i+1} x_{i+6}\right\}_{i \in \mathrm{Z}_{7}}+\xi\left\{x_{i} x_{i-1}^{2} x_{i+2}+x_{i+1}^{2} x_{i-2}\right\}_{i \in \mathrm{Z}_{7}}, \\
V_{4}^{m} & =\left\{x_{i} x_{i+2} x_{i+5}\right\}_{i \in \mathrm{Z}_{7}}, \\
V_{4}^{b} & =\left\{x_{i} x_{i+3} x_{i+4}\right\}_{i \in \mathrm{Z}_{7}} .
\end{aligned}
$$

By (1) we get the following facts:

$$
\begin{aligned}
& \operatorname{Pfaff}\left(M_{7}^{\prime}(x,(1: 0: 0))\right)=\operatorname{Pfaff}(\lambda)=\left\{x_{i} x_{i+3} x_{i+4}\right\}_{i \in \mathrm{Z}_{7}}, \\
& \operatorname{Pfaff}\left(M_{7}^{\prime}(x,(0: 1: 0))\right)=\operatorname{Pfaff}(\mu)=\left\{x_{i} x_{i+1} x_{i+6}\right\}_{i \in \mathrm{Z}_{7}}, \\
& \operatorname{Pfaff}\left(M_{7}^{\prime}(x,(0: 0: 1))\right)=\operatorname{Pfaff}(v)=\left\{x_{i} x_{i+2} x_{i+5}\right\}_{i \in \mathrm{Z}_{7}} .
\end{aligned}
$$


and

$$
\begin{aligned}
& V(\operatorname{Pfaff}(\lambda))=\bigcup_{i \in \mathbf{Z}_{7}}\left\{x_{i}=x_{i+2}=x_{i+5}=0\right\} \cup \bigcup_{i \in \mathbf{Z}_{7}}\left\{x_{i}=x_{i+1}=x_{i+6}=0\right\}, \\
& V(\operatorname{Pfaff}(\mu))=\bigcup_{i \in \mathbf{Z}_{7}}\left\{x_{i}=x_{i+2}=x_{i+5}=0\right\} \cup \bigcup_{i \in \mathbf{Z}_{7}}\left\{x_{i}=x_{i+3}=x_{i+4}=0\right\}, \\
& V(\operatorname{Pfaff}(\nu))=\bigcup_{i \in \mathbf{Z}_{7}}\left\{x_{i}=x_{i+3}=x_{i+4}=0\right\} \cup \bigcup_{i \in \mathbf{Z}_{7}}\left\{x_{i}=x_{i+1}=x_{i+6}=0\right\} .
\end{aligned}
$$

Thus the middle and bottom $V_{4}$ 's are, respectively, Pfaff $(\nu)$ and $\operatorname{Pfaff}(\lambda)$. Therefore, the $\mathrm{P}^{1}$ of degenerations over the cusp $u_{1}=(0: 1: 0: 0) \in$ $\Gamma \subset \mathrm{P}^{3}\left(U^{\prime}\right)$, corresponding to $(0: 1: 0) \in Q \subset \mathrm{P}^{2}(W)$, takes place settheoretically in the following seven projective subspaces

$$
V(\operatorname{Pfaff}(\nu)) \cap V(\operatorname{Pfaff}(\lambda))=\bigcup_{i \in \mathrm{Z}_{7}}\left\{x_{i}=x_{i+1}=x_{i+6}=0\right\} \subset \mathrm{P}^{6}(V) .
$$

The variety $A_{\alpha}$ defined by the above 21 cubics is:

(1) if $\xi \neq 0 \neq \tau$,

$$
A_{\alpha}=\left\{x_{i}=x_{i-1}=x_{i+1}=\xi x_{i+2} x_{i+5}-\tau x_{i+3} x_{i+4}=0\right\}_{i \in \mathrm{Z}_{7}} ;
$$

(2)(a) if $\tau=0$,

$$
A_{\alpha}=\bigcup_{i \in \mathrm{Z}_{7}}\left\{x_{i}=x_{i+1}=x_{i+2}=x_{i+3}=0\right\}
$$

with a double structure;

(b) if $\xi=0$,

$$
\begin{aligned}
A_{\alpha}=\bigcup_{i \in Z_{7}}\left\{x_{i}=x_{i+1}=x_{i+2}=x_{i+4}=0\right\} & \\
\cup & \left\{x_{i}=x_{i+1}=x_{i+2}=x_{i+5}=0\right\} .
\end{aligned}
$$

In terms of the configurations in $\operatorname{VSP}(Q, 6)$ related to these degenerations, and then related to configurations in $H(\Delta)$, we see that $E$, viewed as a subset of $L \subset S^{3} W^{\prime}$, determines via (12) a 4-dimensional vector space of $L^{*}=L \subset$ $S^{3} W$, namely

$$
\{E=0\}=\left\langle y_{1} y_{2} y_{3}, y_{1} y_{2}^{2}, y_{3} y_{1}^{2}, \tau\left(y_{2} y_{3}^{2}\right)+\xi\left(y_{3}^{2} y_{1}-y_{2}^{3}\right)\right\rangle,
$$


and this allows us to recover the matrix $\psi$ in (11):

$$
\psi=\left(\begin{array}{ccc}
-y_{1} & 0 & 0 \\
-\xi y_{2} & y_{3} & 0 \\
\xi y_{3} & 0 & y_{2} \\
\tau y_{3} & -y_{2} & -y_{1}
\end{array}\right)
$$

whose minors are precisely the elements in $\{E=0\}$. Furthermore these minors are the generators of the ideal of the six points defining the (degenerate) element we still call $\zeta$ of $\operatorname{VSP}(Q, 6)$, which is

$$
\zeta=\{(0: 0: 1) \times 2,(1: 0: 0) \times 2,(0: 1:+\sqrt{\xi / \tau}),(0: 1:-\sqrt{\xi / \tau})\} .
$$

Finally we study the three possible cases:

(1) If $\xi \neq 0 \neq \tau$, the embedded point at $(0: 0: 1)$ is a tangent vector along $\left\{\tau y_{2}+\xi y_{1}=0\right\}$, whereas $(1: 0: 0)$ points along $\left\{y_{3}=0\right\}$. Observe that $\zeta$ viewed in $H(\Delta)$ determines a smooth conic in $\left\{u_{1}=0\right\}$ union the line $\left\{u_{2}=u_{3}=0\right\}$.

(2)(a) If $\tau=0$, then $\zeta$ degenerates to two points: the quadruple point $(0: 0: 1)$, whose ring of regular functions is

$$
\mathrm{C}\left[y_{1}, y_{2}\right] /\left(y_{1}^{2}, y_{1} y_{2}, y_{1}-y_{2}^{3}\right) \cong \mathrm{C} \oplus \mathrm{C} y_{2} \oplus \mathrm{C} y_{2}^{2} \oplus \mathrm{C} y_{2}^{3},
$$

which therefore points along $\left\{y_{1}=0\right\}$ with multiplicity 4 , and the double point $(1: 0: 0)$, which points along $\left\{y_{3}=0\right\}$. $\zeta$ viewed in $H(\Delta)$ determines the special smooth conic $\left\{u_{0} u_{2}+u_{3}^{2}=0\right\} \subset\left\{u_{1}=\right.$ $0\}$ union the line $\left\{u_{2}=u_{3}=0\right\}$.

(b) If $\xi=0$, then $(1: 0: 0)$ points along $\left\{y_{3}=0\right\},(0: 1: 0)$ points along $\left\{y_{1}=0\right\}$ and $(0: 0: 1)$ points along $\left\{y_{2}=0\right\} . \zeta$ viewed in $H(\Delta)$ determines the union of $\left\{u_{1}=u_{2}=0\right\},\left\{u_{1}=u_{3}=0\right\}$ and $\left\{u_{3}=u_{2}=0\right\}$ in $\mathrm{P}^{3}(U)$.

Remark 5.17. Case (2a) is the only one where the surface $A_{\alpha}$ is not generated by $V_{7,(0: 0: 1)}$ and $V_{7,(1: 0: 0)}$, in other words by $V_{7, y}$, where $y \in \zeta \in$ $\operatorname{VSP}(Q, 6)$. Notice that Remark 5.6 holds.

REMARK 5.18. The three degenerate elliptic curves corresponding to the cusps $u_{1}, u_{2}$ and $u_{3}$ are the three possible chains of seven projective lines joining two elements of the canonical basis of $\mathrm{P}^{6}(V)$ and invariant under the cyclic action of $Z_{7}$ on the indexes. By the same action we can focus our attention on the projective 3 -spaces $\left\{x_{0}=x_{1}=x_{6}=0\right\}$ where one of the irreducible components lives. 
(1) The general degenerate $(1,7)$-abelian surface over a cusp is given by the union of seven quadric surfaces. The surface on $\left\{x_{0}=x_{1}=x_{6}=0\right\}$ is given by the product of the projective lines through $e_{2}, e_{3}$ and $e_{4}, e_{5}$ shifted by $(\xi: \tau)$, namely $\xi x_{2} x_{5}-\tau x_{3} x_{4}=0$.

(2) When $(\xi: \tau) \rightarrow(1: 0)$ the quadric surface splits into two projective planes, namely $\left\{x_{0}=x_{1}=x_{2}=x_{6}=0\right\}$ and $\left\{x_{0}=x_{1}=x_{5}=x_{6}=0\right\}$. The total of 14 projective planes are divided into 7 pairs of coinciding planes by the cyclic action of $Z_{7}$, hence we get the double structure.

(3) Finally, when $(\xi: \tau) \rightarrow(0: 1)$, the quadric surface again splits into two projective planes, namely $\left\{x_{0}=x_{1}=x_{3}=x_{6}=0\right\}$ and $\left\{x_{0}=x_{1}=\right.$ $\left.x_{4}=x_{6}=0\right\}$. This time the 14 projective planes are all different under the cyclic action of $Z_{7}$. This is the most special degeneration, because it lies on the only point where the fibres over the cusps $u_{1}, u_{2}$ and $u_{3}$ intersect.

Remark 5.19. The result of Proposition 5.16 highlights a difference with the results of $[1]$ on the $(1,5)$ case. The combinatorics in that case tell us that when the quadric surfaces in $\mathrm{P}^{4}$ split up - exactly as in Remark 5.18 - into 10 planes, in both the splits one gets 5 pairs of coinciding planes. This can be seen easily with the corresponding picture and an argument as above. And in fact in the $(1,5)$ case, the multiplicity of the six points over cusps is $(2,2,1,1)$ or $(4,2)$, but not $(2,2,2)$.

\section{Appendix A. Representation theory of $G_{7}$ and $\mathrm{SL}_{2}\left(\mathrm{Z}_{7}\right)$}

Here we follow [16]. As mentioned in Section 2.2, if $V=\mathrm{C}\left(\mathrm{Z}_{7}\right)$, then the Heisenberg group $H_{7}:=H_{1,7}$ is generated by

$$
\sigma\left(x_{i}\right)=x_{i-1}, \quad \tau\left(x_{i}\right)=\xi^{i}\left(x_{i}\right),
$$

where $\xi:=\exp (2 \pi i / 7)$. The Galois group $\Theta$ of $\mathrm{Q}(\xi)$ over Q acts on $H_{7}$ : let $\theta$ be the generator given by $\theta(\xi)=\xi^{3}$. Then $\theta^{3}=$ complex conjugation.

The irreducible $H_{7}$-module $V$ produces five more modules by the composition with the automorphisms $\theta^{i} \in \Theta$. Denote by $V_{i}$ the representation $H_{7} \stackrel{\theta^{i}}{\rightarrow} H_{7} \rightarrow \operatorname{Aut}(V)$. These six representations are inequivalent, as one sees computing their characters, and together with the characters of $Z_{7} \times Z_{7}$ these are all the irreducible characters of $H_{7}$.

We equip $V=\mathrm{C}\left(\mathrm{Z}_{7}\right)$ with the canonical basis $\left\{e_{i}\right\}_{i \in \mathbf{Z}_{7}}$, where $e_{i}(l)=\delta_{i l}$. If $\left\{x_{i}\right\}_{i \in Z_{7}}$ is the dual basis of $V^{\prime}=V_{3}$, then the action of $\sigma$ and $\tau$ on $V$ and on $V^{\prime}=V_{3}=H^{0}(\mathscr{O}(1))$ is given by

$$
\sigma\left(e_{i}\right)=e_{i-1} ; \quad \tau\left(e_{i}\right)=\xi^{i}\left(e_{i}\right): \quad \sigma\left(x_{i}\right)=x_{i-1} ; \quad \tau\left(x_{i}\right)=\xi^{-i}\left(x_{i}\right) .
$$


We recall that $G_{7}:=H_{7} \rtimes\langle\iota\rangle$, where $\iota \in \operatorname{SL}(V), x(i)=-x(-i)$.

\section{A.1. Character table of $G_{7}$}

For each central element $\alpha \in \mu_{7} \subset G_{7}$ we define the conjugacy classes

$$
C_{m, n}=\left\{(\alpha, m, n),(\alpha,-m,-n) \mid \alpha \in \mu_{7}\right\}, \quad(m, n) \neq(0,0),
$$

and

$$
C_{\alpha}=\left\{(\alpha, m, n) \iota \mid m, n \in \mathbf{Z}_{7}\right\} .
$$

There are 7 classes $\{\alpha\}, 24$ classes $C_{m, n}$, each containing 14 elements, and 7 classes $C_{\alpha}$, each containing 49 elements. We denote by $Z$ the sum of all 24 $Z_{s, t}$.

With this notation we get the character table of $G_{7}$ (Table 3), where the column $\star$ gives the corresponding representation.

TABle 3. Character table of $G_{7}$

\begin{tabular}{cccc}
\hline$\{\alpha\}$ & $C_{m, n}$ & $C_{\alpha}$ & $\star$ \\
\hline 1 & 1 & 1 & $I$ \\
$7 \theta^{i}(\alpha)$ & 0 & $\theta^{i}(\alpha)$ & $V_{i}$ \\
1 & 1 & -1 & $S$ \\
$7 \theta^{i}(\alpha)$ & 0 & $-\theta^{i}(\alpha)$ & $V_{i}^{\#}$ \\
2 & $\xi^{s m+t n}+\xi^{-s m-t n}$ & 0 & $Z_{s, t}$ \\
\hline
\end{tabular}

\section{A.2. The group $\mathrm{SL}_{2}\left(\mathrm{Z}_{7}\right)$}

It is convenient to name some elements in $N=H_{7} \rtimes \mathrm{SL}_{2}\left(\mathrm{Z}_{7}\right)$ and their images in $\mathrm{SL}_{2}\left(\mathrm{Z}_{7}\right): \mu: e_{j} \mapsto e_{4 j}$, with $\bar{\mu}=\left(\begin{array}{ll}2 & 0 \\ 0 & 4\end{array}\right) \in \mathrm{SL}_{2}\left(\mathrm{Z}_{7}\right) ; v: e_{j} \mapsto \xi^{j^{2}} e_{j}$, with $\bar{v}=\left(\begin{array}{ll}1 & 0 \\ 2 & 1\end{array}\right) \in \mathrm{SL}_{2}\left(\mathrm{Z}_{7}\right) ; \delta: e_{j} \mapsto \sqrt{\frac{-1}{7}} \sum_{k} \xi^{k j} e_{k}$, with $\bar{\delta}=\left(\begin{array}{rr}0 & -1 \\ 1 & 0\end{array}\right) \in$ $\mathrm{SL}_{2}\left(\mathrm{Z}_{7}\right)$; and $\bar{\varepsilon}=\left(\begin{array}{rr}2 & 2 \\ -2 & 2\end{array}\right) \in \mathrm{SL}_{2}\left(\mathrm{Z}_{7}\right)$.

The conjugacy classes of $\bar{\delta}$ and of $\pm \bar{\varepsilon}$ have 42 elements, those of $\pm \bar{v}$ and $\pm \bar{\nu}^{3}$ have 24, those of $\pm \bar{\mu}$ have 56, and those of \pm id one element each. Observe that $\delta^{2}=\iota$ and that the elements in $\mathrm{SL}_{2}\left(\mathrm{Z}_{7}\right)$ are given according to

$$
\begin{aligned}
& \mu \sigma \mu^{-1}=\sigma^{2} \quad \iota \sigma \iota=\sigma^{-1} \quad \nu \sigma \nu^{-1}=\xi \sigma \tau^{2} \quad \delta \sigma \delta^{-1}=\tau \\
& \mu \tau \mu^{-1}=\tau^{4} \quad \iota \tau \iota=\tau^{-1} \quad \nu \tau \nu^{-1}=\tau \quad \delta \tau \delta^{-1}=\sigma^{-1}
\end{aligned}
$$


In Table 4 we reproduce the character table of $\mathrm{SL}_{2}\left(\mathrm{Z}_{7}\right)$, with the notation $\eta^{ \pm}=(1 \pm \sqrt{-7}) / 2$.

TABLE 4. Character table of $\mathrm{SL}_{2}\left(Z_{7}\right)$

\begin{tabular}{crrrrrrrrrrc}
\hline id & - id & $\bar{\mu}$ & $-\bar{\mu}$ & $\bar{v}$ & $\bar{\nu}^{3}$ & $-\bar{\nu}^{3}$ & $-\bar{v}$ & $\bar{\delta}$ & $\bar{\varepsilon}$ & $-\bar{\varepsilon}$ & $\star$ \\
\hline 1 & 1 & 1 & 1 & 1 & 1 & 1 & 1 & 1 & 1 & 1 & $I$ \\
8 & -8 & -1 & 1 & 1 & 1 & -1 & -1 & 0 & 0 & 0 & $M_{1}$ \\
8 & 8 & -1 & -1 & 1 & 1 & 1 & 1 & 0 & 0 & 0 & $M_{2}$ \\
7 & 7 & 1 & 1 & 0 & 0 & 0 & 0 & -1 & -1 & -1 & $L$ \\
4 & -4 & 1 & -1 & $\eta^{-}$ & $\eta^{+}$ & $-\eta^{+}$ & $-\eta^{-}$ & 0 & 0 & 0 & $U$ \\
4 & -4 & 1 & -1 & $\eta^{+}$ & $\eta^{-}$ & $-\eta^{-}$ & $-\eta^{+}$ & 0 & 0 & 0 & $U^{\prime}=U^{*}$ \\
6 & -6 & 0 & 0 & -1 & -1 & 1 & 1 & 0 & $\sqrt{2}$ & $-\sqrt{2}$ & $T_{1}$ \\
6 & -6 & 0 & 0 & -1 & -1 & 1 & 1 & 0 & $-\sqrt{2}$ & $\sqrt{2}$ & $T_{2}$ \\
6 & -6 & 0 & 0 & -1 & -1 & -1 & -1 & 2 & 0 & 0 & $T$ \\
3 & 3 & 0 & 0 & $-\eta^{+}$ & $-\eta^{-}$ & $-\eta^{-}$ & $-\eta^{+}$ & -1 & 1 & 1 & $W$ \\
3 & 3 & 0 & 0 & $-\eta^{-}$ & $-\eta^{+}$ & $-\eta^{+}$ & $-\eta^{-}$ & -1 & 1 & 1 & $W^{\prime}=W^{*}$ \\
\hline
\end{tabular}

\section{A.3. Decompositions of $\mathrm{SL}_{2}\left(\mathrm{Z}_{7}\right)$ representations}

Consider now the decomposition of $V$ into eigenspaces of $\iota$ as $V=V_{+} \oplus V_{-}$, where

$$
\begin{aligned}
& V_{+}=\operatorname{span}\left\{e_{1}-e_{6}, e_{4}-e_{3}, e_{2}-e_{5}\right\} \\
& V_{-}=\operatorname{span}\left\{2 e_{0}, e_{1}+e_{6}, e_{4}+e_{3}, e_{2}+e_{5}\right\} .
\end{aligned}
$$

Restricting $\mu, v$ and $\delta$ to $V_{+}$and $V_{-}$respectively, one gets:

$$
\begin{aligned}
\mu^{+} & =\left(\begin{array}{lll}
0 & 0 & 1 \\
1 & 0 & 0 \\
0 & 1 & 0
\end{array}\right), \quad \mu^{-}=\left(\begin{array}{cccc}
1 & 0 & 0 & 0 \\
0 & 0 & 0 & 1 \\
0 & 1 & 0 & 0 \\
0 & 0 & 1 & 0
\end{array}\right), \\
\nu^{+} & =\operatorname{diag}\left(\xi, \xi^{4}, \xi^{2}\right), \\
\delta^{+} & =\sqrt{\frac{-1}{7}}\left(\begin{array}{ccc}
\xi-\xi^{6} & \xi^{2}-\xi^{5} & \xi^{4}-\xi^{3} \\
\xi^{2}-\xi^{5} & \xi^{4}-\xi^{3} & \xi-\xi^{6} \\
\xi^{4}-\xi^{3} & \xi-\xi^{6} & \xi^{2}-\xi^{5}
\end{array}\right), \\
\delta^{-} & =\sqrt{\frac{-1}{7}}\left(\begin{array}{cccc}
1 & 1 & 1 & 1 \\
2 & \xi+\xi^{6} & \xi^{2}+\xi^{5} & \xi^{4}+\xi^{3} \\
2 & \xi^{2}+\xi^{5} & \xi^{4}+\xi^{3} & \xi+\xi^{6} \\
2 & \xi^{4}+\xi^{3} & \xi+\xi^{6} & \xi^{2}+\xi^{5}
\end{array}\right) .
\end{aligned}
$$


From the character table of $\mathrm{SL}_{2}\left(\mathrm{Z}_{7}\right)$ one sees that, as a $\mathrm{SL}_{2}\left(\mathrm{Z}_{7}\right)$-module, $V=$ $W^{\prime} \oplus U^{\prime}$ and from the above computations one gets concrete realisations of $W^{\prime}$ and $U^{\prime}$, namely $W^{\prime}=V_{+}$and $U^{\prime}=V_{-}$. Furthermore the following computations play a crucial role:

$$
\begin{array}{llrl}
S^{2} W & =T & & S^{2} W^{\prime}=T \\
S^{3} W & =L \oplus W^{\prime} & & S^{3} W^{\prime}=L \oplus W \\
S^{4} W & =I \oplus M_{2} \oplus T & & S^{4} W^{\prime}=I \oplus M_{2} \oplus T .
\end{array}
$$

If we denote by $v_{1}=e_{1}-e_{6}, v_{2}=e_{4}-e_{3}$ and $v_{3}=e_{2}-e_{5}$ the chosen basis of $W^{\prime}$, then the only $\mathrm{SL}_{2}\left(\mathrm{Z}_{7}\right)$-invariant quartic is the Klein quartic in (21). Notice also that

$$
S^{2} U^{\prime}=L \oplus W^{\prime}
$$

We choose as basis for $L \subset S^{2} U^{\prime}$ the following elements:

$$
\begin{aligned}
& f_{0}=u_{0}^{2}, \quad f_{1}=u_{2} u_{3}, \quad f_{2}=u_{3} u_{1}, \quad f_{3}=u_{1} u_{2}, \\
& f_{4}=u_{0} u_{3}+u_{1}^{2}, \quad f_{5}=u_{0} u_{1}+u_{2}^{2}, \quad f_{6}=u_{0} u_{2}+u_{3}^{2},
\end{aligned}
$$

and as basis for $W^{\prime}$ the elements

$$
v_{2}=u_{0} u_{3}-u_{1}^{2}, \quad v_{3}=u_{0} u_{1}-u_{2}^{2}, \quad v_{1}=u_{0} u_{2}-u_{3}^{2} .
$$

Then in the decomposition $S^{3} V_{3}=\left(I \oplus U^{\prime} \oplus L\right) \otimes V_{4}$ the elements corresponding to $f_{j} e_{0}$ are given by

$$
\begin{array}{lll}
f_{1} e_{0}=x_{0} x_{1} x_{6} & f_{4} e_{0}=x_{2}^{2} x_{3}+x_{5}^{2} x_{4} \\
f_{2} e_{1} x_{2} x_{4}+x_{3} x_{5} x_{6}-x_{0} x_{2} x_{5} & f_{5} e_{0}=x_{4}^{2} x_{6}+x_{3}^{2} x_{1} \\
f_{3} e_{0}=x_{0} x_{3} x_{4} & f_{6} e_{0}=x_{1}^{2} x_{5}+x_{6}^{2} x_{2} .
\end{array}
$$

From here one obtains all $f_{j} e_{k}$ via cyclic permutation, in other words via the action of $\sigma$.

\section{Appendix B. The Klein quartic $Q$}

We think this interesting curve deserves some space in this article, so we give the following presentation that was suggested by Alastair King. We use the notation $(\cdot)^{\prime}$ for the dual of a vector space. The interested reader can find a beautiful and classic treatise on this topic in [13], where most of this material can be found. Notice that all the notation is consistent with A.2 and A.3.

Let $X(7)$ be the abstract modular curve of level 7. It is embedded in the projective plane $\mathrm{P}^{2}(W)$ by the canonical linear system $W^{\prime}=H^{0}(X(7), \omega)$, where $\omega$ is the canonical line bundle. There is a choice of basis $v_{1}, v_{2}, v_{3}$ for 
$W^{\prime}$ so that the homogeneous coordinate vector $\left(v_{1}, v_{2}, v_{3}\right)$ of a point in the concrete model $X(7) \subset \mathrm{P}^{2}(W)$ of $X(7)$ satisfies

$$
f_{\text {Klein }}=v_{1}^{3} v_{2}+v_{2}^{3} v_{3}+v_{3}^{3} v_{1}
$$

The simple group $G=\mathrm{PGL}_{2}\left(\mathrm{Z}_{7}\right)$ of order 168 acts on the Klein quartic as its full automorphism group.

The space $W^{\prime}$ is a 3-dimensional 'fundamental' representation of $G$ : it is faithful, of minimal dimension and all other irreducible representations are contained in spaces of tensors over it.

Note that $W^{\prime}$ is unimodular but not self-dual, i.e. $W \cong \wedge^{2} W^{\prime} ¥ W^{\prime}$, and so it is important to distinguish $W$ and $W^{\prime}$, and consequently $\mathbf{P}^{2}(W)$ and $\mathbf{P}^{2}\left(W^{\prime}\right)$. In particular, $\mathrm{P}^{2}\left(W^{\prime}\right)$ also contains a unique $G$-invariant quartic $X(7)^{\prime}$, which is isomorphic to $X(7)$ as an abstract curve, but not as a curve with $G$-action.

$\mathrm{PGL}_{2}\left(\mathrm{Z}_{7}\right)$ has a 4-dimensional projective representation $U=H^{0}(X(7)$, $\left.\omega^{3 / 2}\right)$. In other words, $U$ is a representation of the central extension $\widehat{G}=$ $\mathrm{SL}_{2}\left(\mathrm{Z}_{7}\right)$ in which the centre \pm 1 acts non-trivially. It is necessary to pass to this central extension to lift the $G$-action to the chosen square root $\omega^{1 / 2}$ of the canonical bundle. In the case of $X(7)$ there is a natural choice (see [13], Section 9). The representation $U$ is also unimodular and is also not self-dual.

We give some results we use in the article. Consider the kernel $R$ of the multiplication map

$$
U \otimes W^{\prime} \rightarrow H^{0}\left(X(7), \omega^{5 / 2}\right) .
$$

Taking a certain basis $a_{0}, a_{1}, a_{2}, a_{3}$ for $U$, the kernel $R$ has a basis of 'bilinear relations'

$$
\begin{aligned}
v_{1} a_{1}+v_{2} a_{2}+v_{3} a_{3} & =0 \\
v_{1} a_{0}-v_{3} a_{1} & =0 \\
v_{2} a_{0}-v_{1} a_{2} & =0 \\
v_{3} a_{0}-v_{2} a_{3} & =0
\end{aligned}
$$

which can be written either as

$$
\left(\begin{array}{llll}
a_{0} & a_{1} & a_{2} & a_{3}
\end{array}\right)\left(\begin{array}{cccc}
0 & v_{1} & v_{2} & v_{3} \\
v_{1} & -v_{3} & 0 & 0 \\
v_{2} & 0 & -v_{1} & 0 \\
v_{3} & 0 & 0 & -v_{2}
\end{array}\right)=0,
$$

or

$$
\left(\begin{array}{lll}
v_{1} & v_{2} & v_{3}
\end{array}\right)\left(\begin{array}{cccc}
a_{1} & a_{0} & -a_{2} & 0 \\
a_{2} & 0 & a_{0} & -a_{3} \\
a_{3} & -a_{1} & 0 & a_{0}
\end{array}\right)=0
$$


Thus the consistency conditions for the bilinear system (22) are

$$
\operatorname{det}\left(\begin{array}{cccc}
0 & v_{1} & v_{2} & v_{3} \\
v_{1} & -v_{3} & 0 & 0 \\
v_{2} & 0 & -v_{1} & 0 \\
v_{3} & 0 & 0 & -v_{2}
\end{array}\right)=-\left(v_{1}^{3} v_{2}+v_{2}^{3} v_{3}+v_{3}^{3} v_{1}\right)=0,
$$

and

$$
\operatorname{rank}\left(\begin{array}{cccc}
a_{1} & a_{0} & -a_{2} & 0 \\
a_{2} & 0 & a_{0} & -a_{3} \\
a_{3} & -a_{1} & 0 & a_{0}
\end{array}\right) \leq 2
$$

i.e. the system of cubics (4), .,,(7).

This latter system gives the equations satisfied by the embedding of $X(7)$ in the projective 3 -space $\mathrm{P}^{3}\left(U^{\prime}\right)$ by the linear system $U$. In this article we have given this concrete curve in $\mathrm{P}^{3}\left(U^{\prime}\right)$ the name $\Gamma$.

Note further that the bilinear system (22) also implies a further system of equations

$$
\left(\begin{array}{cccc}
a_{1} & a_{0} & -a_{2} & 0 \\
a_{2} & 0 & a_{0} & -a_{3} \\
a_{3} & -a_{1} & 0 & a_{0}
\end{array}\right)\left(\begin{array}{cccc}
0 & -v_{2} v_{3} & -v_{3} v_{1} & -v_{1} v_{2} \\
v_{3} v_{2} & 0 & v_{1}^{2} & -v_{3}^{2} \\
v_{1} v_{3} & -v_{1}^{2} & 0 & v_{2}^{2} \\
v_{1} v_{2} & v_{3}^{2} & -v_{2}^{2} & 0
\end{array}\right)=0
$$

These 12 equations are a basis for the kernel of the multiplication map

$$
U \otimes S^{2} W^{\prime} \rightarrow H^{0}\left(X(7), \omega^{7 / 2}\right) .
$$

The Pfaffian of the second matrix in (27) is simply the Klein quartic equation (21) again. This matrix also reflects the isomorphism

$$
S^{2} W \cong \Lambda^{2} U^{\prime} \subset \operatorname{Hom}\left(U, U^{\prime}\right) .
$$

In [13] the system (26) and most of (22) are worked out via another relationship between $U$ and $W^{\prime}$, namely the isomorphism

$$
S^{2} U \cong H^{0}\left(X(7), \omega^{3}\right) \cong S^{3} W^{\prime} .
$$

This is expressed explicitly by the system of equations in (13) which make up the summand $L$, while the three others in (14) make up the summand $W$.

Note that the right hand sides in (14) are the derivatives of the Klein quartic. Combinations of the last three equations in (13) together with those in (14) 
give expressions for the various other monomials:

$$
\begin{aligned}
& a_{0} a_{1}=v_{1}^{2} v_{2} \quad a_{0} a_{2}=v_{2}^{2} v_{3} \quad a_{0} a_{3}=v_{3}^{2} v_{1} \\
& a_{2}^{2}=-\left(v_{1}^{2} v_{2}+v_{3}^{3}\right) \quad a_{3}^{2}=-\left(v_{2}^{2} v_{3}+v_{1}^{3}\right) \quad a_{1}^{2}=-\left(v_{3}^{2} v_{1}+v_{2}^{3}\right) \\
& v_{3}^{3}=-\left(a_{0} a_{1}+a_{2}^{2}\right) \quad v_{1}^{3}=-\left(a_{0} a_{2}+a_{3}^{2}\right) \quad v_{2}^{3}=-\left(a_{0} a_{3}+a_{1}^{2}\right)
\end{aligned}
$$

These equations easily imply three of the four equations in (22) in the following form which Klein records in [13], Equation (43).

$$
\frac{a_{1}}{a_{0}}=\frac{v_{1}}{v_{3}} \quad \frac{a_{2}}{a_{0}}=\frac{v_{2}}{v_{1}} \quad \frac{a_{3}}{a_{0}}=\frac{v_{3}}{v_{2}}
$$

The remaining equation is then effectively the equation of the quartic itself, which follows by computing e.g. $\left(a_{1} a_{2}\right)^{2}$ in two different ways. Note that the system (28) cannot follow directly from (22) because the latter is unchanged by independent rescaling of the variables, while the system above determines $\left(a_{0}, a_{1}, a_{2}, a_{3}\right)$ from $\left(v_{1}, v_{2}, v_{3}\right)$ up to a sign. However, (22) does imply (28) up to a single overall constant of proportionality.

\section{REFERENCES}

1. Barth, W., Hulek, K., and Moore, R., Degenerations of Horrocks-Mumford surfaces, Math. Ann. 277 (1987), 735-755.

2. Ciliberto, C., and Hulek, K., A remark on the geometry of elliptic scrolls and bielliptic surfaces, Manuscripta Math. 95 (1998), 213-224.

3. Drezet, J. -M., Fibrés exceptionels et variétés des modules de faisceaux semi-stables sur $\mathrm{P}_{2}(\mathrm{C})$, J. Reine Angew. Math. 380 (1987), 14-58.

4. Eisenbud, D., Commutative Algebra with a View toward Algebraic Geometry, Grad. Texts in Math. 150 (1995).

5. Ellingsrud, G., Piene, R., and Strømme, S. A., On the variety of nets of quadrics defining twisted cubics, in: F. Ghione et al, Eds., Space curves (Rocca di Papa, 1985), 84-96: Lecture Notes in Math. 1266 (1987).

6. Fulton, W., Algebraic Curves, W. A. Benjamin, New York 1969.

7. Gross, M., and Popescu, S., Equations of $(1, d)$-polarized abelian surfaces, Math. Ann. 310 (1998), 333-377.

8. Gross, M., and Popescu, S., Calabi-Yau threefolds and moduli of abelian surfaces I, Compositio Math. 127 (2001), 169-228.

9. Harris, J., Algebraic Geometry, Grad. Texts in Math. 133 (1992).

10. Horrocks, G., and Mumford, D., A rank 2 vector bundle on $\mathrm{P}^{4}$ with 15000 symmetries, Topology 12 (1973), 63-81.

11. Hulek, K., Kahn, C., and Weintraub, S. H., Moduli Spaces of Abelian Surfaces: Compactification, Degenerations, and Theta Functions, de Gruyter Exp. Math. 12 (1993).

12. Hulek, K., and Sankaran, G. K., The geometry of Siegel modular varieties, in: S. Mori and Y. Miyaoka (Eds.), Higher Dimensional Birational Geometry, 89-156: Adv. Stud. Pure Math. 35, Kinokuniya, Tokyo 2002.

13. Klein, F., Über die Transformation siebenter Ordnung der elliptischen Funktionen, Math. Ann. 14 (1879), 428-471. 
14. Lange, H., and Birkenhake, C., Complex Abelian Varieties, Grundlehren Math. Wiss. 302 (1992).

15. Levy, S., The eightfold way, Math. Sci. Res. Inst. Publ. 35 (1999).

16. Manolache, N., and Schreyer, F. -O., Moduli of (1, 7)-polarized abelian surfaces via syzygies, Math. Nachr. 226 (2001), 177-203.

17. Marini, A., On the degenerations of $(1,7)$-polarised abelian surfaces, $\mathrm{Ph} . \mathrm{D}$. thesis, University of Bath, 2002.

18. Melliez, F., and Ranestad, K., Degenerations of $(1,7)$-polarized abelian surfaces, preprint math.AG/0309306, 2003.

19. Mukai, S., Fano 3-folds, in: G. Ellingsrud et al, Eds., Complex projective geometry (Trieste, 1989/Bergen, 1989), 255-263: London Math. Soc. Lecture Note Ser. 179 (1992).

20. Newstead, P. E., Introduction to moduli problems and orbit spaces, Tata Inst. Fund. Res. Lectures on Math. and Phys. 51 (1978).

21. Schreyer, F. -O., Geometry and algebra of prime Fano 3-folds of genus 12, Compositio Math. 127 (2001), 297-319.

22. Shafarevich, I. R., Basic Algebraic Geometry, Grundlehren Math. Wiss. 213 (1974).

DIPARTIMENTO DI MATEMATICA E INFORMATICA

UNIVERSITÀ DI UDINE

VIA DELLE SCIENZE

33100 UDINE

ITALY

E-mail: alfio_marini@yahoo.it 